\title{
Compact reconfigurable kirigami
}

\author{
Gary P. T. Choi $\odot,{ }^{1}$ Levi H. Dudte, ${ }^{2}$ and L. Mahadevan $\odot^{2,3}$ \\ ${ }^{1}$ Department of Mathematics, Massachusetts Institute of Technology, Cambridge, Massachusetts 02139, USA \\ ${ }^{2}$ School of Engineering and Applied Sciences, Harvard University, Cambridge, Massachusetts 02138, USA \\ ${ }^{3}$ Departments of Physics, and Organismic and Evolutionary Biology, Harvard University, Cambridge, Massachusetts 02138, USA
}

(Received 13 February 2021; accepted 27 August 2021; published 13 October 2021)

\begin{abstract}
Kirigami involves cutting a flat, thin sheet that allows it to morph from a closed, compact configuration into an open deployed structure via coordinated rotations of the internal tiles. By recognizing and generalizing the geometric constraints that enable this art form, we propose a design framework for compact reconfigurable kirigami patterns, which can morph from a closed and compact configuration into a deployed state conforming to any prescribed target shape, and subsequently be contracted into a different closed and compact configuration. We further establish a condition for producing kirigami patterns and mechanisms which are reconfigurable and rigid deployable allowing us to connect the compact states via a zero-energy family of deployed states. All together, our inverse design framework lays out a path for the creation of shape-morphing material structures.
\end{abstract}

DOI: 10.1103/PhysRevResearch.3.043030

\section{INTRODUCTION}

Kirigami is the art of using cuts in a single sheet of paper that allow for changes in the shape of the sheet via coordinated rotations of the connected facets. In recent years, the art form has motivated the design of metamaterials wherein architected cuts on a flat, thin sheet of material can lead to unusual properties not found in most naturally occurring materials, such as a negative Poisson's ratio [1]. There have been a vast number of studies on the geometry, topology, and mechanics of kirigami [2-8] with applications to the design of nanocomposites [9,10], shape-morphing sheets [11,12], inflatable structures [13], soft robots [14], etc. Almost without exception, the deployed kirigami structures are open and periodic, and the property of admitting multiple closed and compact contracted states has been addressed only in a few well-known periodic regular kirigami patterns [15-17], and lead to states that are related to each other via global rotations. This raises a natural question: Is it possible to introduce cuts on a thin sheet of material in a way that yields a deployable and reconfigurable kirigami structure conforming to any prescribed shape and admitting multiple closed and compact contracted states? Here we answer this question in the affirmative by building on our recent inverse design framework [6] and identifying a set of geometric constraints to achieve both reconfigurability and a range of different energy landscapes associated with the deployment pathway. This allows us to design kirigami patterns that can undergo certain nontrivial shape changes under deployment to achieve a prescribed de-

Published by the American Physical Society under the terms of the Creative Commons Attribution 4.0 International license. Further distribution of this work must maintain attribution to the author $(s)$ and the published article's title, journal citation, and DOI. ployed or contracted shape, while also being reconfigurable and rigid deployable.

Since kirigami derives its properties via coordinated rotations of the individual cells, it is natural that the problem of kirigami design is close to the design of mechanisms [18-22], in which a set of moving parts are connected by kinematic joints to form a deployable structure. However, we note that while kinematic mechanism designs mainly focus on rigid-deployable structures formed by rigid bars, our inverse kirigami framework is applicable to both rigid and stretchable materials. Furthermore, by focusing on how the angles and edge lengths of the tiles in a given kirigami pattern can be changed to achieve the desired properties, we circumvent the role of designing structural topology for mechanism design.

\section{RECONFIGURABLE KIRIGAMI DESIGN}

To crystallize our question in a minimal setting, we consider the quad kirigami patterns, a class of deployable structures obtained by introducing cuts to form quadrilateral tiles connected at hinges [see Fig. 1(a)] with a single global degree of freedom. To determine the size and orientation of the cuts that yield a deployed configuration approximating a prescribed target shape, it is more convenient to work in the deployed space and change the geometry of each tile. In our recent work [6], we have shown that the key criteria for guaranteeing that the deformed deployed configuration yields a valid kirigami pattern, also known as the contractibility constraints, consist of the edge length constraints and the angle sum constraints. As illustrated by the red dotted lines in Fig. 1(a), the edge length constraints enforce the constancy of the length of each pair of edges in the deployed space that correspond to the same edge in the pattern space:

$$
a=b, \quad c=d, \quad p=q, \quad r=s .
$$

The angle sum constraints enforce the condition that the set of angles $\left\{\alpha_{k}\right\}_{k=1}^{n}$ (for quad kirigami, $n=4$ ) in the deployed 
(a)

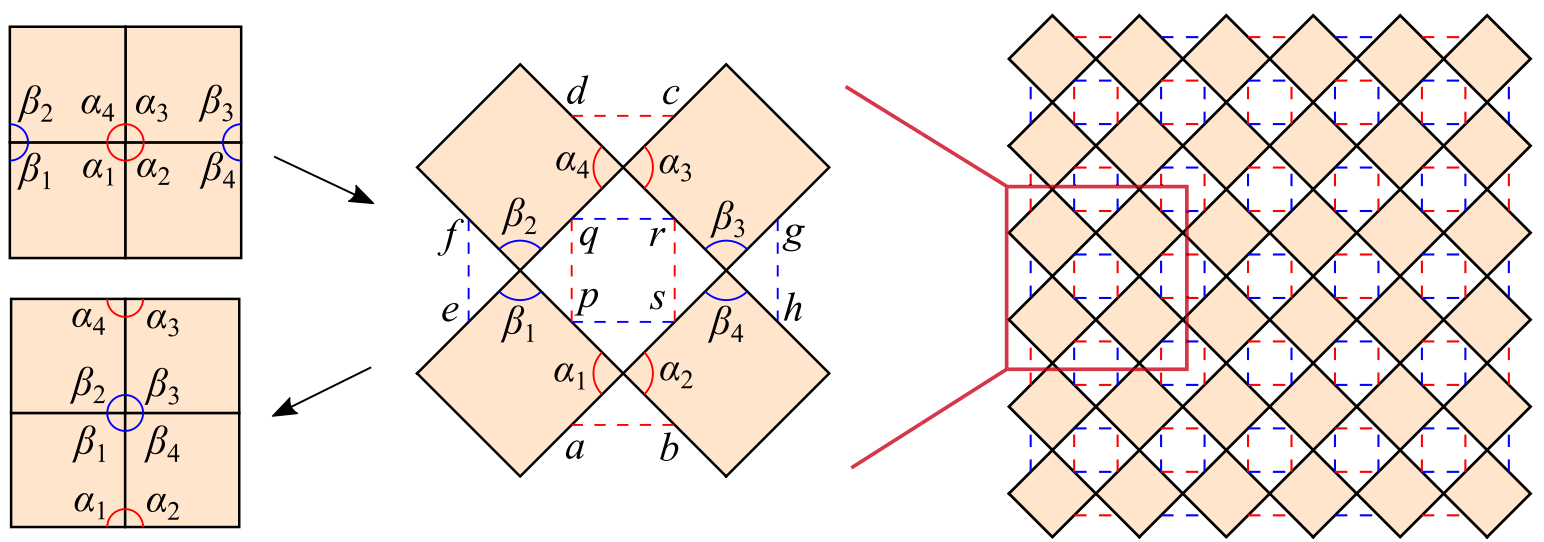

(b)

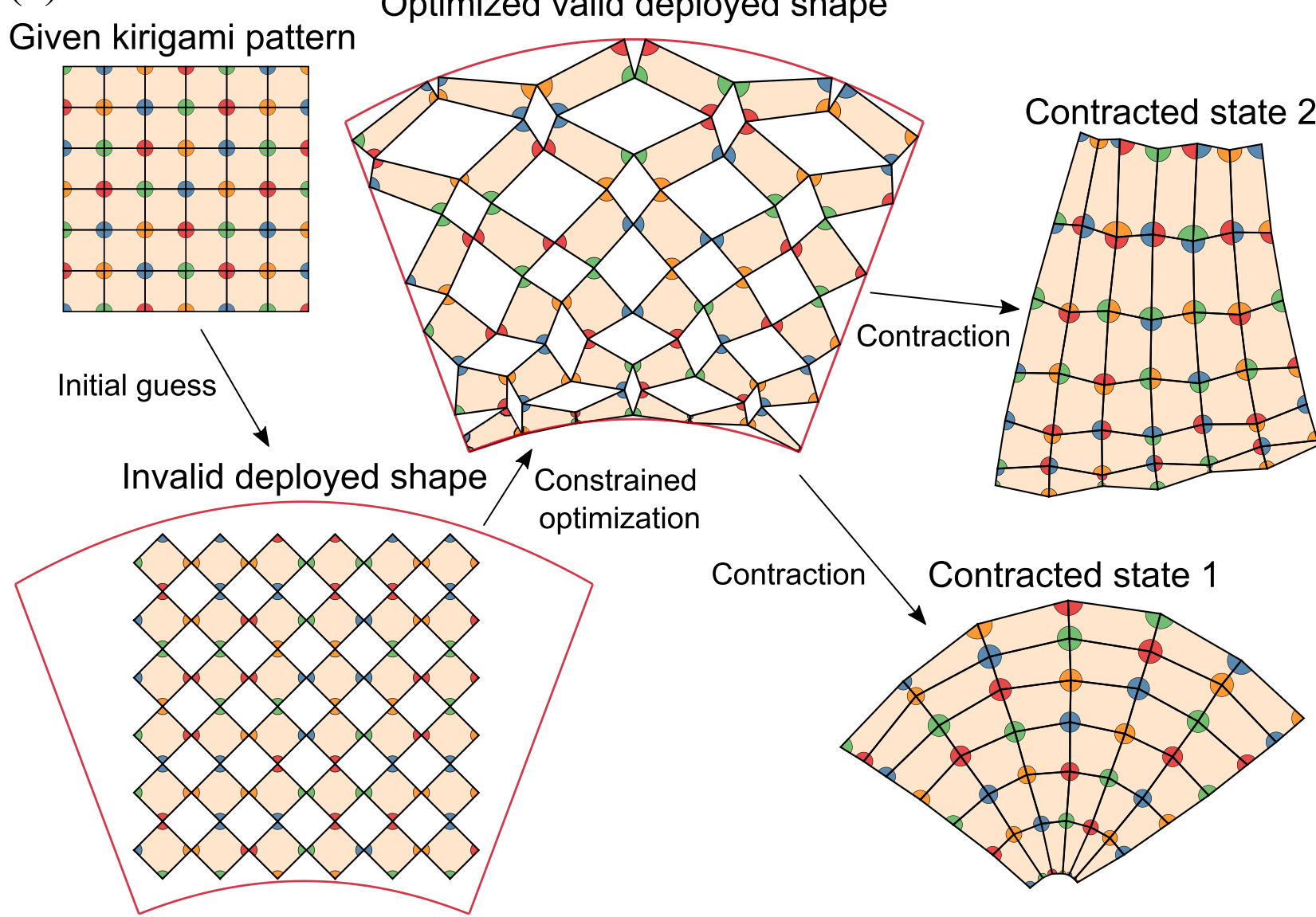

FIG. 1. Reconfigurable kirigami design. (a) An enlargement of the unit cell of a quad kirigami tessellation illustrating the constraints on edge lengths and angles to be satisfied [Eqs. (1)-(4)]. The red dotted lines indicate the ordinary edge pairs corresponding to the same cuts, and the blue dotted lines indicate the dual edge pairs for getting the other contracted configuration. (b) The inverse design framework for reconfigurable kirigami. Starting with a given kirigami pattern and a prescribed target shape, we construct an initial guess in the deployed space and solve a constrained optimization problem to obtain a valid deployed configuration that satisfies both the ordinary contractibility constraints, and the new reconfigurability constraints, and matches the target shape. We then contract the deployed configuration in two ways, one by following the cut edge pairs and one by following the dual edge pairs to obtain two contracted states. The angles are colored based on the correspondence in the kirigami pattern.

space that correspond to the same vertex in the pattern space [see Fig. 1(a)] satisfy

$$
\sum_{k=1}^{n} \alpha_{k}=2 \pi
$$

To ensure that the deformed deployed configuration admits another closed and compact contracted state, we exploit an underlying duality present in the standard quad kirigami pattern associated with reconfigurability, which implies the presence of dual pairs of length and angle constraints. These read as follows: 


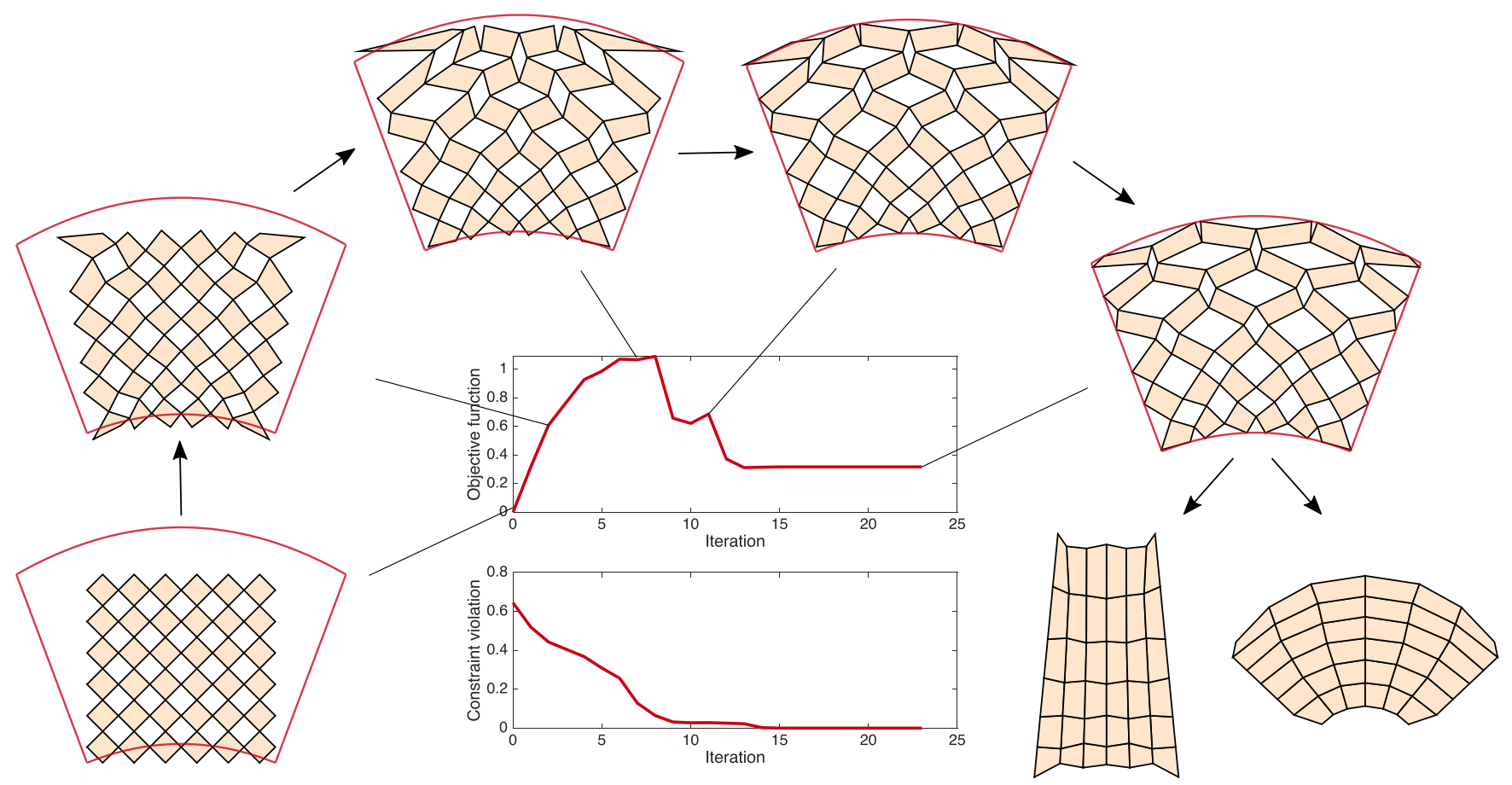

FIG. 2. The optimization process for producing a reconfigurable kirigami pattern. The two plots show the objective function [Eq. (7)] and the maximum constraint violation, which takes all constraints in Eqs. (1)-(6) into consideration. We use MATLAB's fmincon optimization solver to iteratively update the coordinates of the nodes and change the pattern from the initial guess to an optimal configuration that matches the target shape and satisfies the reconfigurability conditions. As the pattern is reconfigurable, it admits two different closed and compact contracted states.

(1) Dual edge length constraints. For each pair of adjacent edges belonging to two different tiles and not paired up in Eq. (1) [i.e., the blue dotted lines in Fig. 1(a)], we should have

$$
e=f, \quad g=h, \quad p=s, \quad q=r,
$$

noting that each pair of them will then correspond to the same edge in the reconfigured pattern space.

(2) Dual angle sum constraints. For every set of angles $\left\{\beta_{k}\right\}_{k=1}^{n}$ dual to the set of angles $\left\{\alpha_{k}\right\}_{k=1}^{n}$ in Eq. (2) inside a unit cell [Fig. 1(a)], we should have

$$
\sum_{k=1}^{n} \beta_{k}=2 \pi
$$

noting that $\beta_{1}, \beta_{2}, \ldots, \beta_{n}$ will then correspond to the same vertex in the reconfigured pattern space.

Altogether, for quad kirigami, the contractibility constraints proposed in [6] and the reconfigurability constraints proposed in this work enforce that all edges around each hole in the deployed configuration must be equal in length, yielding a rhombus, and that all angles of the deformed tiles at two opposite corners of each rhombus hole should add up to $2 \pi$.

In addition to the internal constraints, to further ensure that the deployed configuration conforms to a prescribed boundary shape, we must also enforce the shape matching constraints [6] for every boundary node $\mathbf{p}_{i}$ :

$$
\left\|\mathbf{p}_{i}-\widetilde{\mathbf{p}}_{i}\right\|^{2}=0,
$$

where $\widetilde{\mathbf{p}}_{i}$ is the projection of $\mathbf{p}_{i}$ onto the target boundary.
Given the above constraints, we are now in a position to frame the inverse design framework for reconfigurable kirigami, shown in Fig. 1(b). Given a regular kirigami tessellation and a prescribed target shape, we start with an initial guess of the deployed configuration, which can either be a trivial deployment of the standard tessellation, a deformed configuration produced by a conformal/quasiconformal map $[24,25]$, or any other methods that preserve the number and connectedness of the tiles. Almost without exception, any initial guess will violate at least some of the constraints in contractibility, reconfigurability, or target shape matching. To obtain a valid reconfigurable kirigami pattern, we formulate a constrained optimization problem for the deployed configuration with all constraints above together with the nonoverlap constraints which prevent adjacent tiles from overlapping. At every angle between two adjacent faces in the deployed space, we enforce the following inequality [6]:

$$
\langle(\mathbf{b}-\mathbf{a}) \times(\mathbf{c}-\mathbf{a}), \vec{n}\rangle \geqslant 0,
$$

where $\mathbf{a}$ and $\mathbf{b}$ are two nodes of a face, $\mathbf{a}$ and $\mathbf{c}$ are two nodes of another face, $(\mathbf{b}, \mathbf{a}, \mathbf{c})$ form a right-hand ordered angle between the two faces, and $\vec{n}=(0,0,1)$ is the outward unit normal. Here we simply adopt the objective function used in our previous work [6] to yield a smooth shape change over the entire pattern, noting that other choices are possible:

$$
\min \frac{1}{M} \sum_{i=1}^{M}\left(\sum_{j}\left(\alpha_{i_{j}}-\beta_{i_{j}}\right)^{2}+\sum_{k}\left(a_{i_{k}}-b_{i_{k}}\right)^{2}\right),
$$


(a)

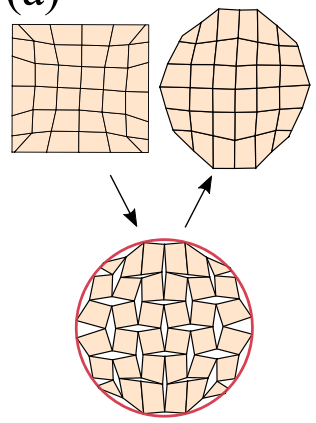

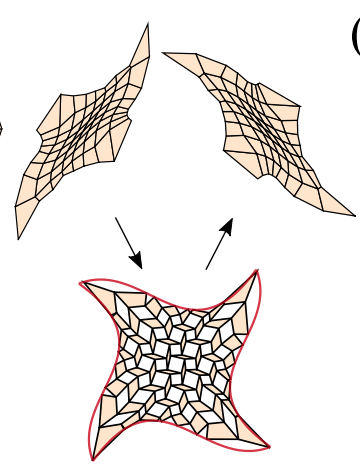

(b)
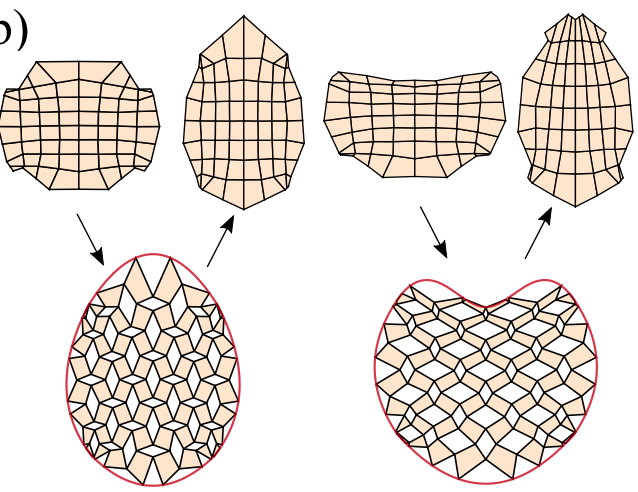

(c)

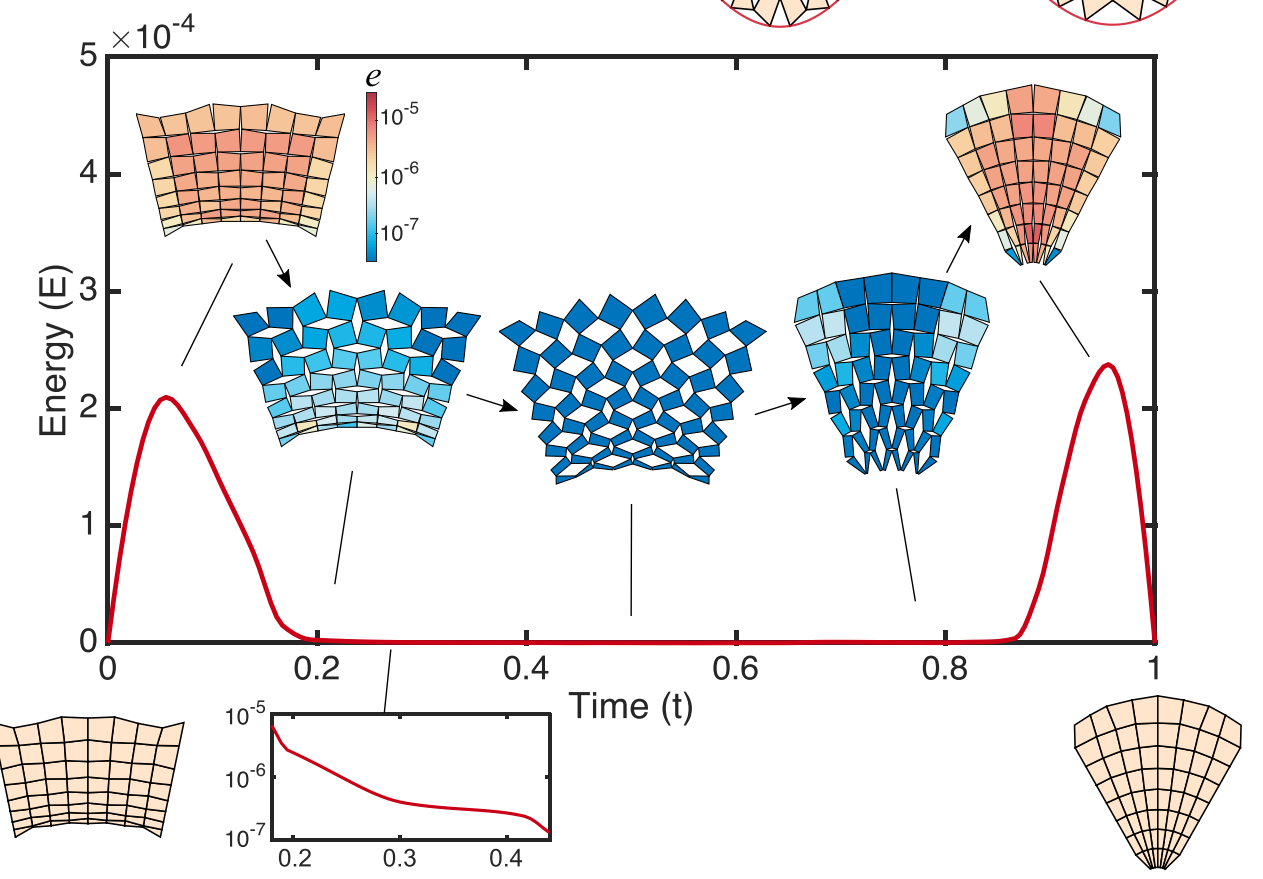

FIG. 3. Reconfigurable kirigami patterns. (a) For each example, the top row shows the two contracted states and the bottom row shows the deployed state which matches the prescribed target boundary shape [see Fig. 4(a) for more examples]. (b) Reconfigurable kirigami patterns produced by further enforcing a reflection symmetry constraint in the constrained optimization framework [see Fig. 4(b) for more examples]. (c) Energetics of the deployment and contraction of a reconfigurable kirigami pattern shows barriers near the two contracted states, but almost zero energy in between. This results in an unusual landscape, with monostable elastic minima at the ends and a mechanism-like zero-energy phase in between (see also the inset log-scale plot). The insets show the intermediate deployed states with each tile color coded by the total spring energy along all its edges and diagonals (denoted by $e$ ) (see Video S1 [23] and Appendix A for details).

where $\alpha_{i_{j}}, \beta_{i_{j}}$ are a pair of corresponding angles in two adjacent cells and $a_{i_{k}}, b_{i_{k}}$ are corresponding edge lengths in two adjacent cells, and $M$ is the total number of pairs of adjacent cells.

Expressing all constraints and the objective function in terms of the coordinates of the nodes in the deployed configuration, we solve this optimization problem using the fmincon routine in MATLAB, where the gradients of the objective function and the constraints are computed analytically and specified in the solver using the SpecifyObjectiveGradient and SpecifyConstraintGradient options. This yields a deformed deployed configuration that satisfies all constraints, from which we can obtain the two contracted states by rotating the tiles according to the two sets of edge correspondences.

Figure 2 shows an example of the optimization process by the MATLAB's fmincon solver. In this example, the target deployed shape is a rainbow and we start with the standard deployed configuration of a quad kirigami pattern as the initial guess. As all adjacent cells are identical, the initial value of the objective function is 0 . However, since the target shape is not matched, the initial constraint violation is large. Throughout the iterations, the solver gradually updates the coordinates of the nodes to match the target shape, which results in a difference in angle and length between adjacent cells and hence the objective function value becomes nonzero. As the optimization process continues, the solver minimizes the objective function and the constraint violation. Finally, it reaches an optimal solution, a valid deployed configuration of a reconfigurable kirigami pattern that satisfies the target deployed shape and admits two closed and compact contracted states. Figure 3(a) shows several examples of reconfigurable kirigami patterns obtained by our method, where each of the kirigami patterns admits two distinct contracted states and the deployed configuration conforms to a prescribed 
(a)
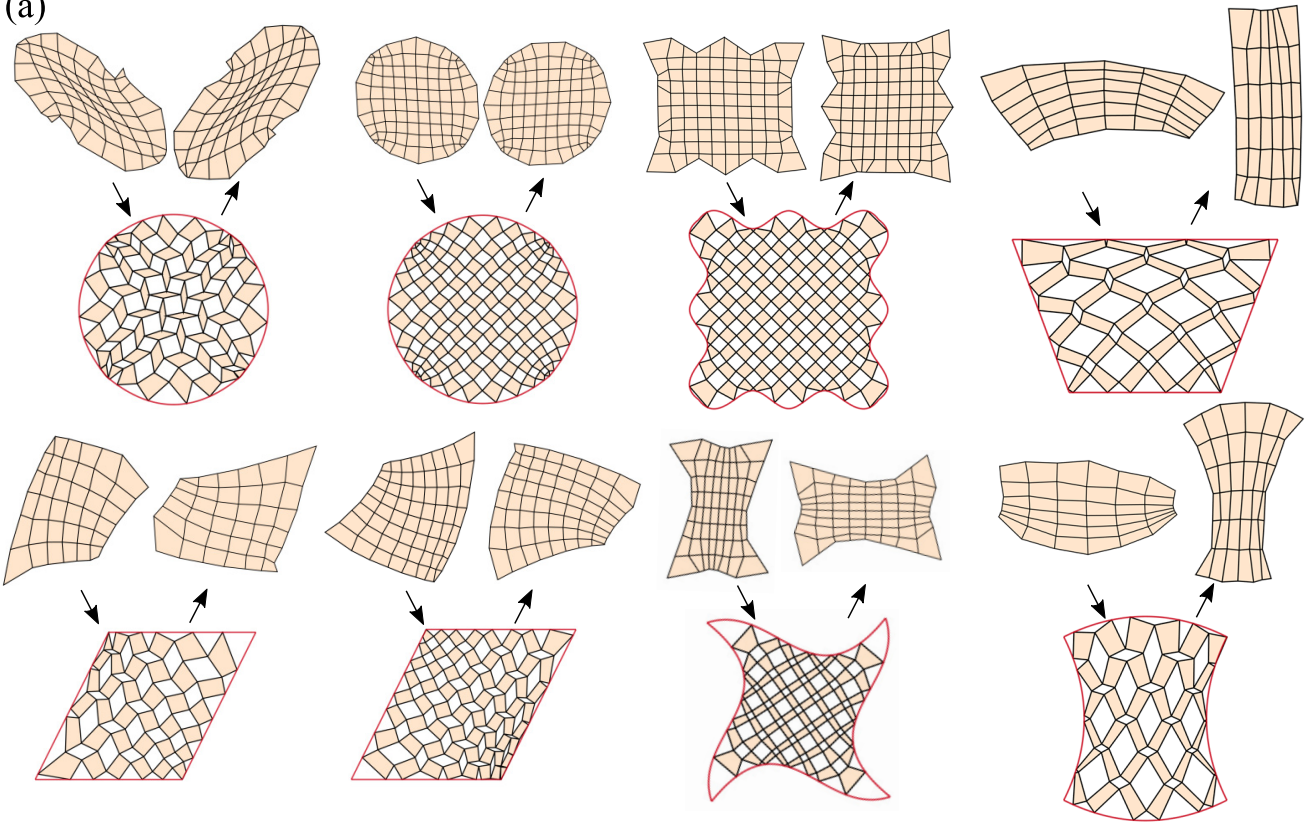

(b)
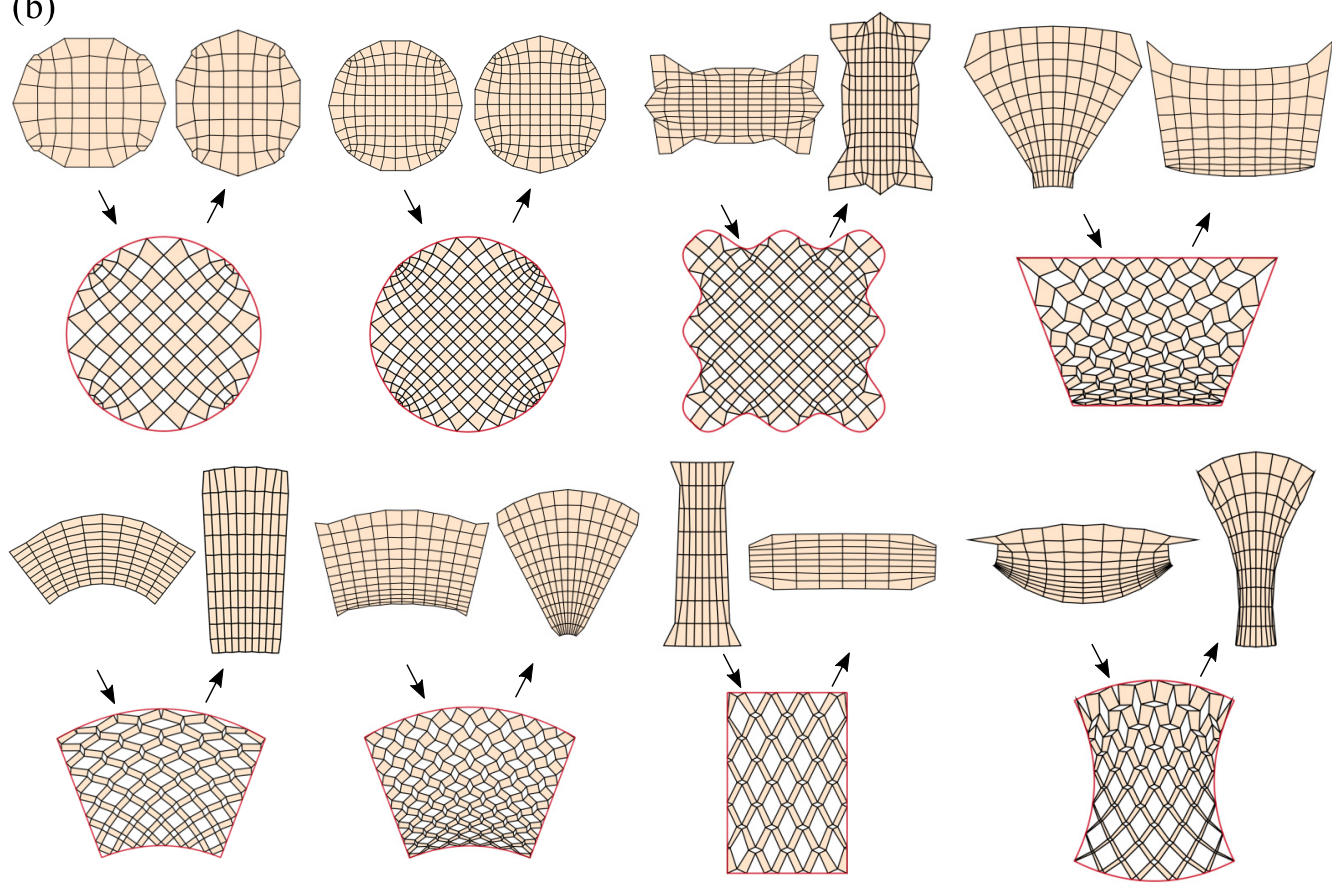

FIG. 4. More examples of reconfigurable quad kirigami patterns with different target boundary shape obtained by our framework. (a) Patterns produced without enforcing any symmetry constraint. For each example, the two closed and compact contracted configurations and the deployed configuration matching the prescribed target shape are shown. Prescribing a symmetric target boundary shape does not necessarily yield a symmetric pattern. (b) With an additional left-right symmetry constraint enforced in the constrained optimization framework, the patterns produced will be symmetric.

intermediate target shape. We see that our method is capable of approximating target shapes with different curvature properties, like our previous inverse design framework [6]. Additionally, we can also control the boundary shape of a contracted state by introducing additional constraints on the boundary edge lengths and angles, yielding a reconfigurable kirigami pattern that deploys from a contracted rectangle to a circle and then contracts to another shape. Of particular interest is the fact that it is possible to use microscopic tile rotations that induce local topological rearrangements to induce an effective overall global rotation. In addition, if the deployed target shape is symmetric, one can further enforce this as an additional constraint in the optimization framework to produce reconfigurable kirigami patterns that are symmetric in the contracted and deployed states [Fig. 3(b)].

The presence of multiple closed and compact contracted states in these reconfigurable structures naturally implies the presence of at least a bistable mechanical energy landscape 
(a)

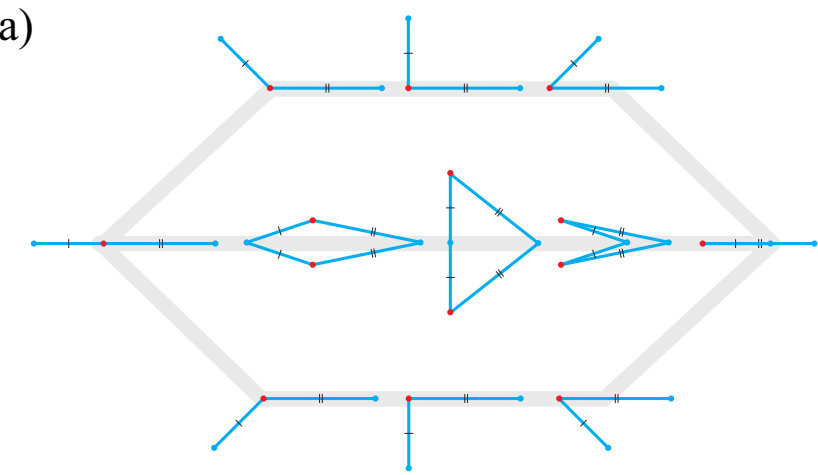

(b)

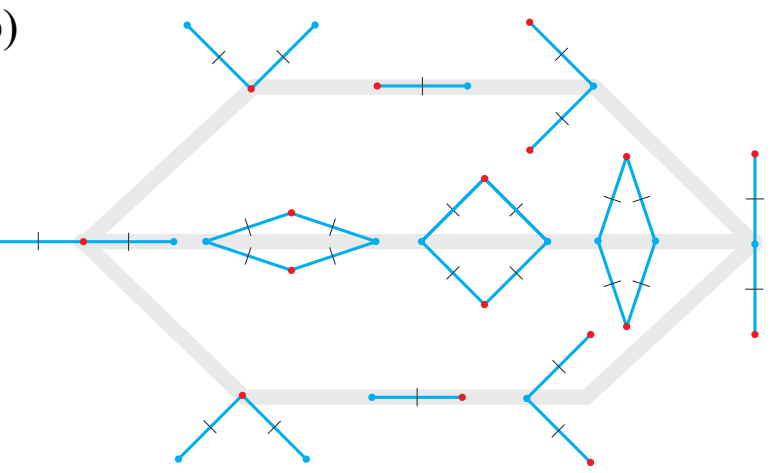

FIG. 5. Conditions for multiple closed, compact contracted kirigami states can be mapped to linkage deployment. (a) The deployment of a generic four-bar linkage. The grey lines show the three deployment paths, two of which (the top and bottom rows) are degenerate. As for the nondegenerate one (the middle row), since the edges are not necessarily equal in length, it is not possible to achieve multiple closed and compact contracted states. (b) The deployment of a reconfigurable four-bar linkage. The grey lines show the three deployment paths, two of which (the top and bottom rows) are degenerate. As for the nondegenerate one (the middle row), note that the edges are equal in length and hence it is possible to achieve multiple closed and compact contracted states.

[Fig. 3(c)], with additional minima arising as a function of new constraints. To characterize this energy landscape, we quantify the deformation of the tiles by replacing the edges and diagonals of the quads by linear springs with a total energy

$$
E(t)=\sum_{i, j:[i, j] \in \mathcal{E}}\left(\frac{\left\|\mathbf{x}_{i}(t)-\mathbf{x}_{j}(t)\right\|-l_{i j}}{l_{i j}}\right)^{2},
$$

where $\mathcal{E}$ is the set of all edges and diagonals, $\mathbf{x}_{i}(t)$ is the position of the node $i$ at time $t$, and $l_{i j}$ is the rest length of the spring at $[i, j]$ (see Appendix A for more details). When we calculate the elastic energy as a function of the deployment stage, we find that the two compact end states are indeed global energy minima, but the tiles have to deformed to move away from these states. Interestingly, there is a regime of deployment where the system is more like a mechanism with the tiles essentially responding by just rotating [see Fig. 3(c) inset and Video S1 of the Supplemental Material [23]]. This is a generic feature of reconfigurable kirigami patterns that behave like elastic structures near their compact states, and like rigid mechanisms away from them.
More reconfigurable kirigami patterns are given in Fig. 4. As shown in Fig. 4(a), the inverse design method may produce asymmetric patterns even if the target boundary shape is symmetric. Figure 4(b) shows examples of symmetric reconfigurable quad kirigami patterns produced by enforcing an additional left-right symmetry constraint in the constrained optimization process. More specifically, we first shift the target pattern such that the symmetry axis is $x=0$. We then make only the left half nodes (with $x<0$ ) as variables [in the form of $\left.\left(x_{i}, y_{i}\right), i=1,2, \ldots\right]$, while each of the corresponding nodes in the right half is enforced to be $\left(-x_{i}, y_{i}\right)$. The coordinates are then used in the optimization process as usual for determining the constraint violation and descent direction. It can be observed that with this additional constraint, the results are more regular in shape. Note that reconfigurable kirigami patterns that approximate a prescribed shape are not necessarily unique. Using different initial guesses, it is possible to obtain different reconfigurable kirigami patterns. The large variety of shapes produced demonstrate the flexibility of our framework.

One can also consider each negative space in a quad kirigami pattern as a four-bar linkage to analyze reconfigurability. Figure 5 shows the deployments of a generic four-bar linkage and a reconfigurable four-bar linkage respectively. As illustrated in the middle row of Fig. 5(a), for a generic $2 \times 2$ quad kirigami pattern, it is not possible to morph the pattern from a closed and compact contracted configuration to a deployed state and then contract it to another closed and compact contracted configuration while remaining embedded in two dimensions. By contrast, as illustrated in the middle row of Fig. 5(b), for a reconfigurable $2 \times 2$ quad kirigami pattern, all four edges of the negative space are equal in length and hence it is possible to achieve multiple closed and compact contracted configurations.

\section{RECONFIGURABLE AND RIGID-DEPLOYABLE KIRIGAMI DESIGN}

Our previous example shows that generic quad kirigami patterns are not single degree-of-freedom mechanisms [6], and thus neither are reconfigurable kirigami patterns that we have introduced so far. While one may control the stability of the patterns by tuning the hinge stiffness as discussed in [6], it is noteworthy that the hinge stiffness is not considered in the constrained optimization problem and hence has to be handled separately. This raises a natural question: How can we complement the geometric constraints in Eqs. (3) and (4) to preserve the single degree of freedom in the kirigami patterns by modifying the proposed inverse design framework without introducing extra steps? Said differently, how can we make quad kirigami rigid-deployable by admitting a single continuous path from one contracted pattern through the solved deployed state to the second contracted pattern state such that all the constituent tiles rotate rigidly without deforming? To enable this, we introduce the following rigid-deployability constraints: around every negative space (i.e., a hole formed by four edges of four neighboring quads), we should have

$$
\alpha_{1}+\alpha_{2}=\alpha_{3}+\alpha_{4}=\beta_{1}+\beta_{2}=\beta_{3}+\beta_{4}=\pi,
$$

where the design angles are as shown in Fig. 1. Intuitively, note that a four-bar linkage has two one-dimensional rigid 


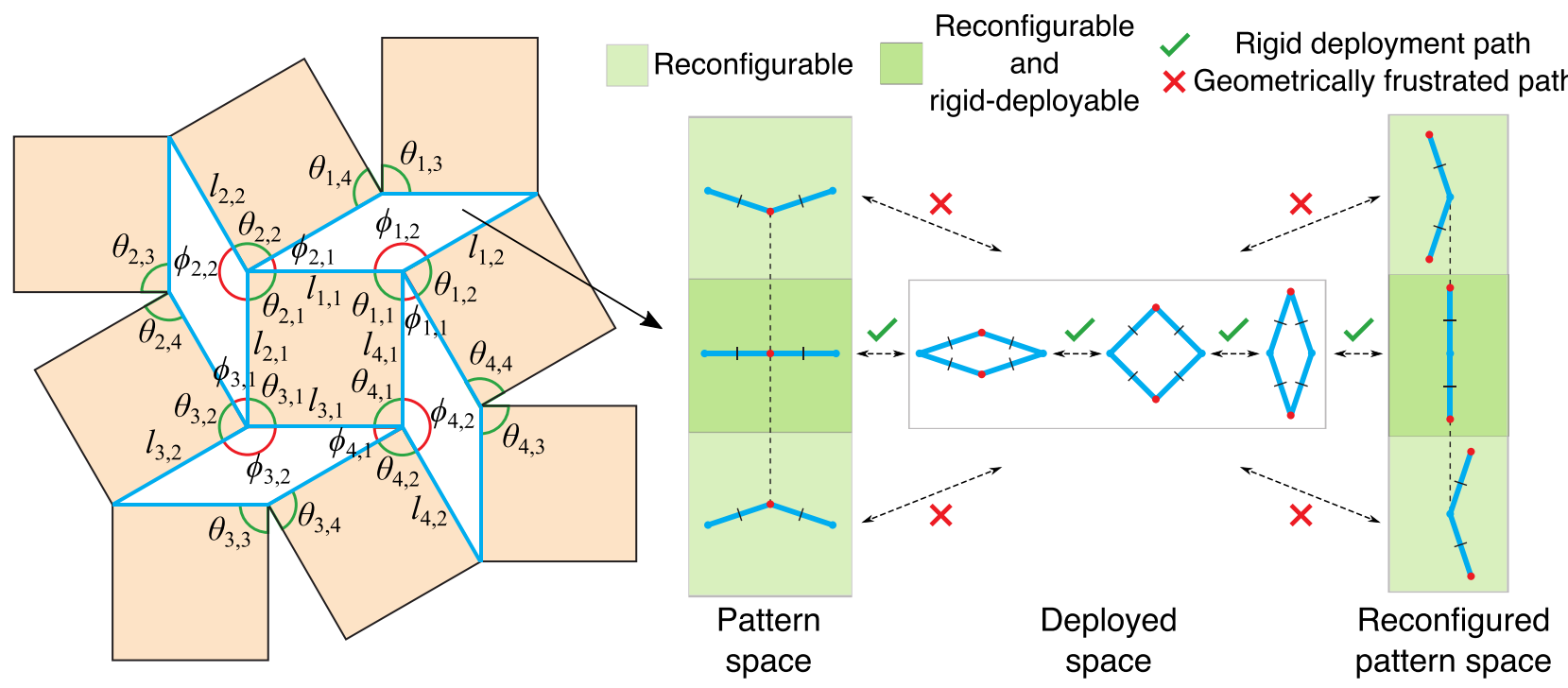

FIG. 6. An illustration of the constraints for reconfigurable, rigid-deployable kirigami design. Left: In kirigami patterns, each negative space (blue) formed by a generic deployed quad kirigami structure (not necessarily reconfigurable) is a four-bar linkage with two pairs of adjacent edges having the same length. Right: If the reconfigurability constraints are enforced, all links in the four-bar linkage have the same length. Such a linkage has three rigid deployments (shown by the dotted lines), one nontrivial path in which all angles between links are activated (the horizontal dotted lines with arrows in between the two dark green boxes in the pattern space and the reconfigured pattern space) and two degenerate paths connected by branch points at the ends of the first path (the vertical dotted lines in the green boxes in the pattern space and the reconfigured pattern space). The linkage can deploy rigidly from the branch point into either deployment paths, but cannot rigidly transform directly between points on the deployment paths while remaining embedded in two dimensions. If Eq. (9) is satisfied, the four-bar linkage in the pattern space will be as shown in the dark green box, and hence there is a rigid deployment path (as indicated by the green ticks) for morphing it from the pattern space to the deployed space and then to the reconfigured pattern space. However, if Eq. (9) is not satisfied, the four-bar linkage in the pattern space will be as shown in the pale green boxes, and so there is no rigid deployment path for reconfiguring it (as indicated by the red crosses).

deployments connected by a single branch point, the configuration with all edges collinear and an angle of $\pi$ between overlapping edge pairs at the common hinge (see the top and bottom rows of Figs. 5(a) and 5(b)). The linkage can deploy rigidly from the branch point into either deployment paths, but cannot rigidly transform directly between points on the deployment paths while remaining embedded in two dimensions. Therefore, a reconfigurable quad kirigami pattern is rigid-deployable if and only if all edges of each negative space are collinear in the contracted configuration. A mathematical justification of the constraints stated in Eq. (9) is provided below.

Lemma. (Local rigid deployability) A reconfigurable kirigami pattern is locally rigid-deployable if and only if Eq. (9) is satisfied for all negative spaces.

Proof. Equation (9) ensures that each negative space forms a straight line in both contracted configurations. Taken in isolation, each negative space can be thought of as a fourbar linkage (highlighted in blue in Fig. 6, left). A negative space/four-bar linkage from a generic quad kirigami pattern (i.e., one that is not reconfigurable) has two unique edge lengths where edges with equal lengths are incident to each other [see Fig. 5(a)]. Such a four-bar linkage has two one-dimensional deployment paths in the plane connected to each other at two branch points, where the edges with equal lengths coincide with each other and all edges are collinear. In the plane, the four-bar linkage cannot move from one deployment path to another except at and through a branch point. Thus, quad kirigami patterns which do not satisfy the rigid-deployability constraints contain negative spaces which cannot pass from pattern to deployed states in the plane without changing edge lengths. And, conversely, quad kirigami patterns which satisfy the rigid-deployability constraints have only negative spaces which can rigidly deform from their straight-line pattern configurations to their solved, deployed configurations in the plane. Reconfigurable quad kirigami structures have negative spaces/four-bar linkages with all lengths being equal [see Fig. 5(b)]. Such linkages have three one-dimensional deployment paths, one path in which all hinges are activated and the linkage forms a rhombus, and two degenerate paths in which two of the four hinges in the linkage are activated, each connected to the rhombus path at a respective branch point (Fig. 6, right). Thus, reconfigurable quad kirigami patterns satisfying Eq. (9) have only negative spaces which can rigidly deform from their two straight-line pattern configurations to their solved, deployed configurations in the plane and hence are locally rigid deployable. And thus if Eq. (9) is violated for some negative space in a reconfigurable quad kirigami pattern, the pattern cannot be locally rigid deployable as the four-bar linkage cannot rigidly move to a branch point while remaining embedded in two dimensions.

We now prove the following result:

Theorem 1. (Global rigid deployability) A reconfigurable kirigami pattern is globally rigid-deployable if and only if Eq. (9) is satisfied for all negative spaces. 
(a)

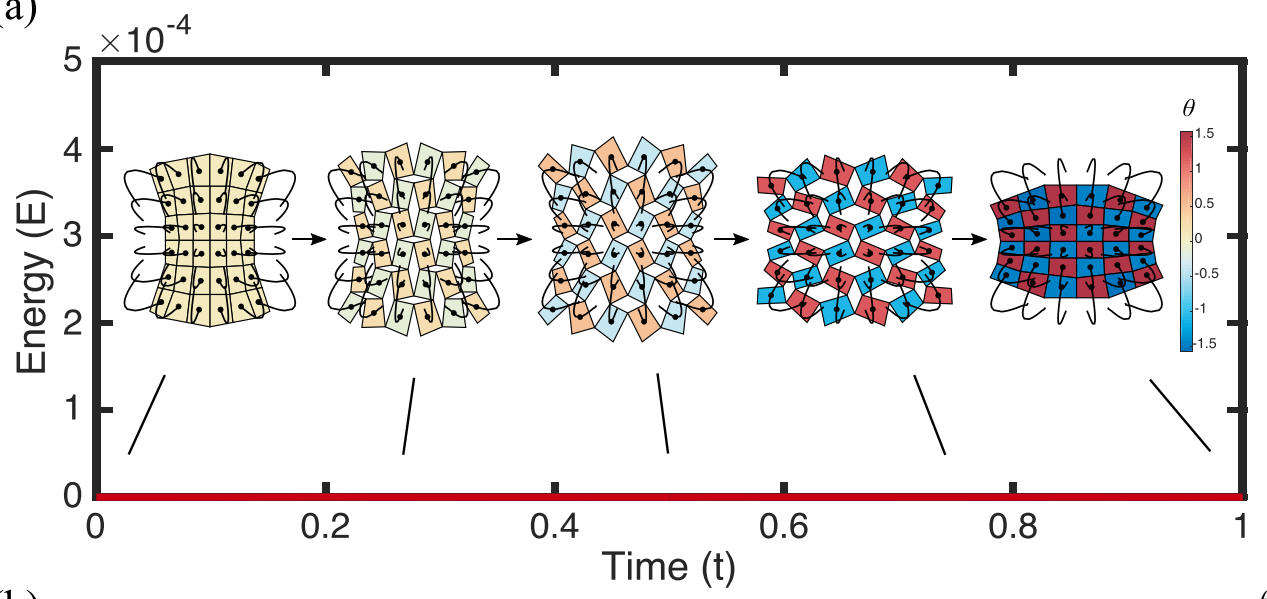

(b)
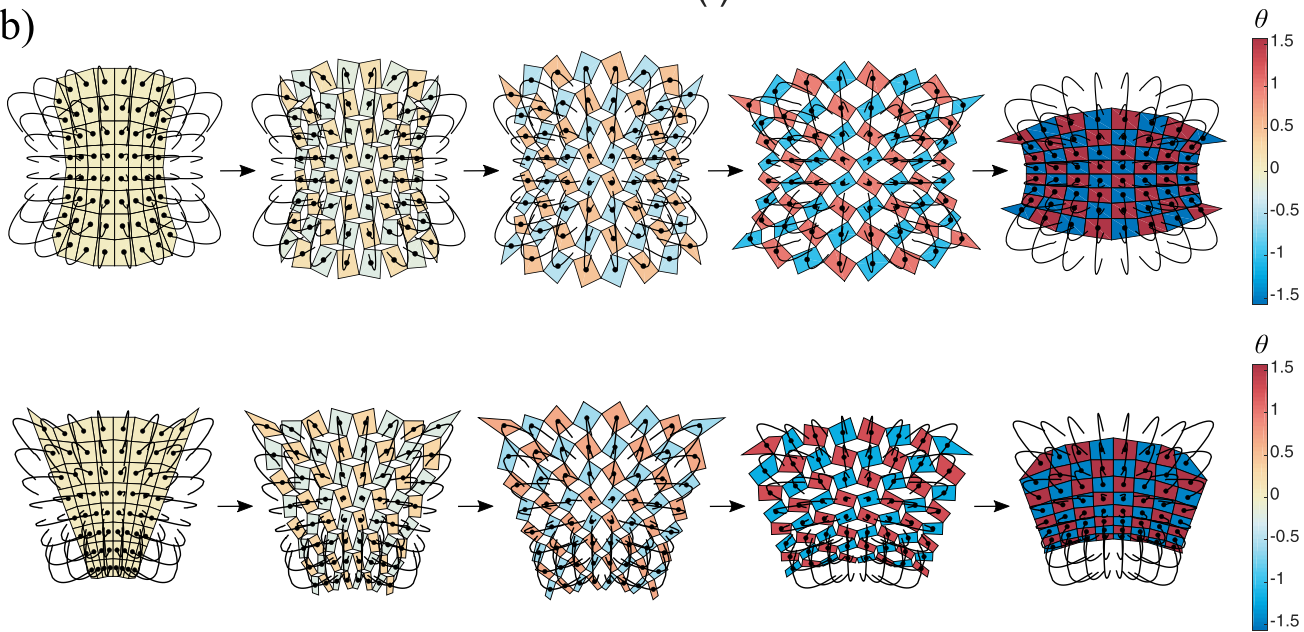

(c)

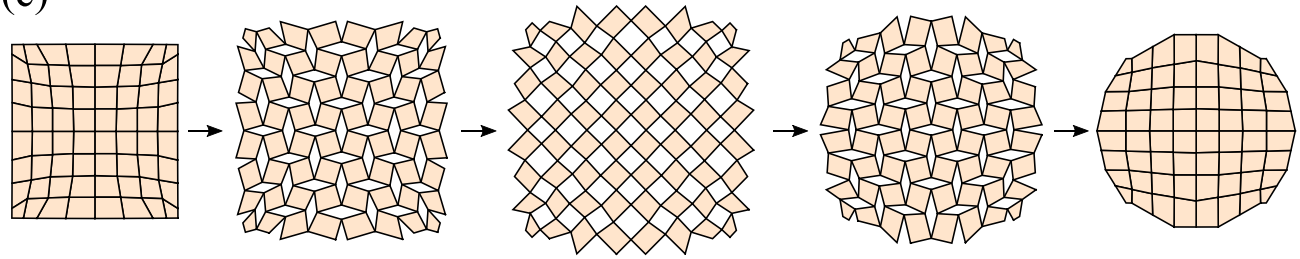

FIG. 7. Reconfigurable, rigid-deployable kirigami patterns. (a) The deployment of a reconfigurable and rigid-deployable kirigami pattern at different states [see also Video S2 [23] and Fig. 8(a)]. The rigid-deployability constraints lead to a flat energy landscape, which indicates that the tiles do not deform throughout the process. Each black curve shows the trajectory of a tile center, with the black dot indicating its current position. The color of each tile indicates the orientation change $(\theta)$ with respect to the initial contracted state. (b) The deployment of two other reconfigurable and rigid-deployable kirigami patterns (see Fig. 9 for more examples). (c) Transforming the square to a circle [see also Video S3 [23] and Figs. 8(b) and 10 for more examples].

Proof. The above lemma provides local rigid deployability if and only if the constraints in Eq. (9) are satisfied for each negative space in the pattern. To analyze global rigid deployability, we construct a loop condition $F$ around a single interior face in a generic (i.e., not necessarily reconfigurable) quad kirigami which must be identity at all points along a rigid deployment. As shown in Fig. 6, let $\theta_{i, j}$ be design angles and $\phi_{i, j}$ be deployment angles in a quad kirigami four-bar linkage negative space. Let $f_{i}$ be the function that transfers a deployment angle $\phi_{i, 1}$ to the deployment angle $\phi_{i+1,1}$ by composing angle-sum transfer $h_{i}$ and four-bar kinematics transfer $f_{i}$ such that

$$
\phi_{i+1,1}=f_{i}\left(\phi_{i, 1}\right)=g_{i}\left[h_{i}\left(\phi_{i, 1}\right)\right],
$$

$$
\begin{gathered}
\phi_{i, 2}=h_{i}\left(\phi_{i, 1}\right)=2 \pi-\phi_{i, 1}-\theta_{i, 1}-\theta_{i, 2}, \\
\phi_{i+1,1}=g_{i}\left(\phi_{i, 2}\right) \\
=2 \sin ^{-1}\left(\frac{l_{i, 2} \sin \phi_{i, 2}}{\sqrt{l_{i, 1}^{2}+l_{i, 2}^{2}-2 l_{i, 1} l_{i, 2} \cos \phi_{i, 2}}}\right) .
\end{gathered}
$$

If the loop condition

$$
F\left(\phi_{1,1}\right)=f_{4}\left(f_{3}\left(f_{2}\left(f_{1}\left(\left(\phi_{1,1}\right)\right)\right)\right)\right)=\phi_{1,1}
$$

is satisfied for every value of $\phi_{1,1} \in\left[0,2 \pi-\theta_{1,1}-\theta_{1,2}\right]$ for every interior quad, then the quad kirigami pattern is 
(a)
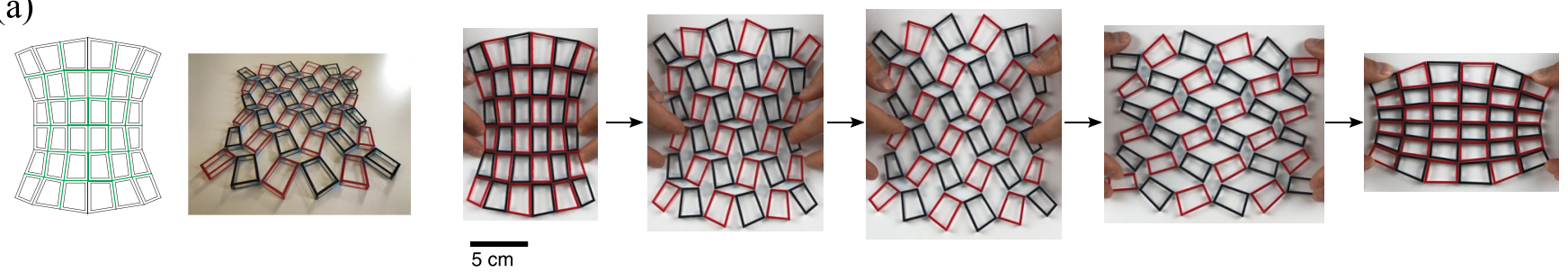

(b)
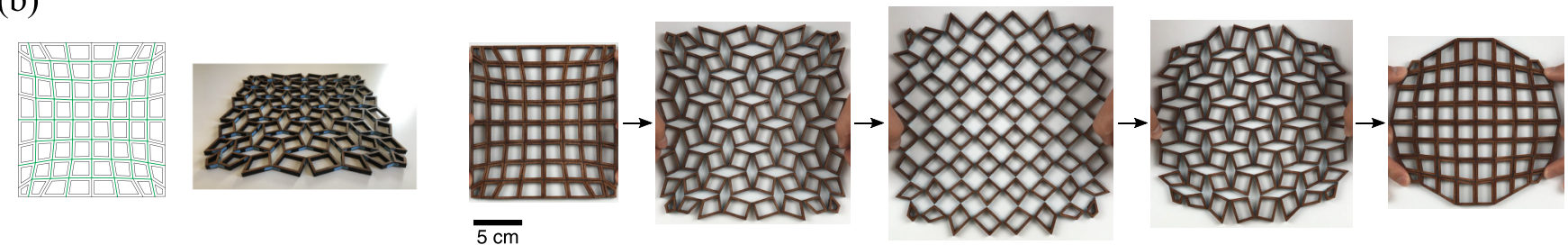

FIG. 8. Physical models of reconfigurable, rigid-deployable kirigami patterns. (a) A physical model for the pattern in Fig. 7(a) cut from a clear acrylic sheet, with the tops/bottoms of pieces checkerboard colored red and black with permanent marker. (b) A physical model for the square-to-circle example in Fig. 7(c) cut from a multiply sheet. For each example, the green edges shown in the leftmost panel form the internal four-bar linkages and black edges complete the quad geometries. Quad interiors have been removed to reduce the weight of the models. Once the pieces have been laser cut, hinges are formed by applying small strips of light blue tape across pairs of vertical faces incident to the hinge vertex. The second left panel shows the perspective view of the assembled model. The right panel shows snapshots of the physical model at different states (see also Videos S4 and S5 [23]). All tiles in the physical model are rigid and undergo no deformation throughout the deployment, while the thickness of the tape joints has some minor effect at the two contracted states and so the model needs to be held still with a small external force at the end states.

globally rigid-deployable. In a reconfigurable quad kirigami pattern, we have $\theta_{i, 1}+\theta_{i, 2}=\pi$ and $l_{i, 1}=l_{i, 2}$ and hence $\phi_{i, 2}=h_{i}\left(\phi_{i, 1}\right)=\pi-\phi_{i, 1}, \phi_{i+1,1}=g_{i}\left(\phi_{i, 2}\right)=$ $\pi-\phi_{i, 2}, \phi_{i+1,1}=f_{i}\left(\phi_{i, 1}\right)=\phi_{i, 1}$. So $F$ is a composition of identity functions $f_{i}$ and is itself identity. Thus, reconfigurable quad kirigami patterns satisfying Eq. (9) are globally rigid-deployable.

Therefore, we can obtain reconfigurable kirigami patterns which are (globally) rigid-deployable by simply augmenting our constrained optimization framework with the additional condition (9). In other words, the constrained optimization now involves the original contractibility constraints [Eqs. (1) and (2)], the reconfigurability constraints [Eqs. (3) and (4)], the shape matching constraints, the nonoverlap constraints, as well as the rigid-deployability constraints [Eq. (9)]. As a remark, by considering the tiles as rigid parts, our method can also be used for designing reconfigurable mechanisms.

Figure 7(a) shows a reconfigurable, rigid-deployable kirigami pattern obtained by our method. In contrast to the patterns in Fig. 3, here each four-bar linkage in the rigiddeployable pattern forms a pair of straight lines in both contracted states, and hence there is no geometrical frustration throughout the deployment. The trajectory of the tiles throughout the deployment and the zero energy associated with deployment process confirms that the pattern morphs smoothly from a contracted configuration to a deployed configuration and subsequently to another contracted configuration (see also Video S2 [23]). It is noteworthy that the reconfigurability constraints and the rigid-deployability constraints imply that all negative space rhombi are similar (i.e., congruent up to rescaling). Therefore, the change in the orientation of the tiles forms a checkerboard pattern with a magnitude that is spatially uniform and changes continuously from 0 to $\pi / 2$ throughout the deployment and contraction.
Figure 7(b) shows the deployment of two other reconfigurable, rigid-deployable kirigami patterns. It can again be observed that each pattern transforms smoothly from one contracted configuration to another contracted configuration, and the orientation change of the tiles forms a checkerboard pattern. It is also possible to perform the constrained optimization directly on the two contracted configurations without caring about the intermediate states (see Appendix B). As a striking example of this approach, we revisit the question of circling the square [6] via a reconfigurable, rigid-deployable kirigami pattern, with the result shown in Fig. 7(c) (see also Video S3 [23]). Note that a major difference between the pattern in Fig. 7(c) and the one in Fig. 3(a) is that here the circle shape is achieved at the reconfigured contracted state, while in Fig. 3(a) the circle shape is achieved at the deployed state (see Appendix $\mathrm{C}$ for more analyses of the reconfigurable kirigami patterns and other variations).

To realize our computations in a physical setting, we fabricated the model in Fig. 7(a) by laser cutting acrylic plastic sheets and connecting the tiles with tape joints [see Fig. 8(a)], from which we can see that the physical model behaves just as predicted. We also fabricated another physical model made of laser-cut wooden tiles connected with tapes for the square-to-circle pattern in Fig. 7(c) [see Fig. 8(b)]. The results demonstrate the flexibility of our proposed reconfigurable kirigami design framework which yields geometric constructions that are material independent (see also Appendix D).

Figure 9(a) shows an example of reconfigurable, rigiddeployable kirigami patterns at different resolutions, with their deployed shapes approximating the same circle. By increasing the cut resolution, a higher accuracy of the approximation of the prescribed shape can be achieved. Figure 9(b) shows more examples of reconfigurable, rigid-deployable kirigami patterns. In particular, note that we can obtain a 

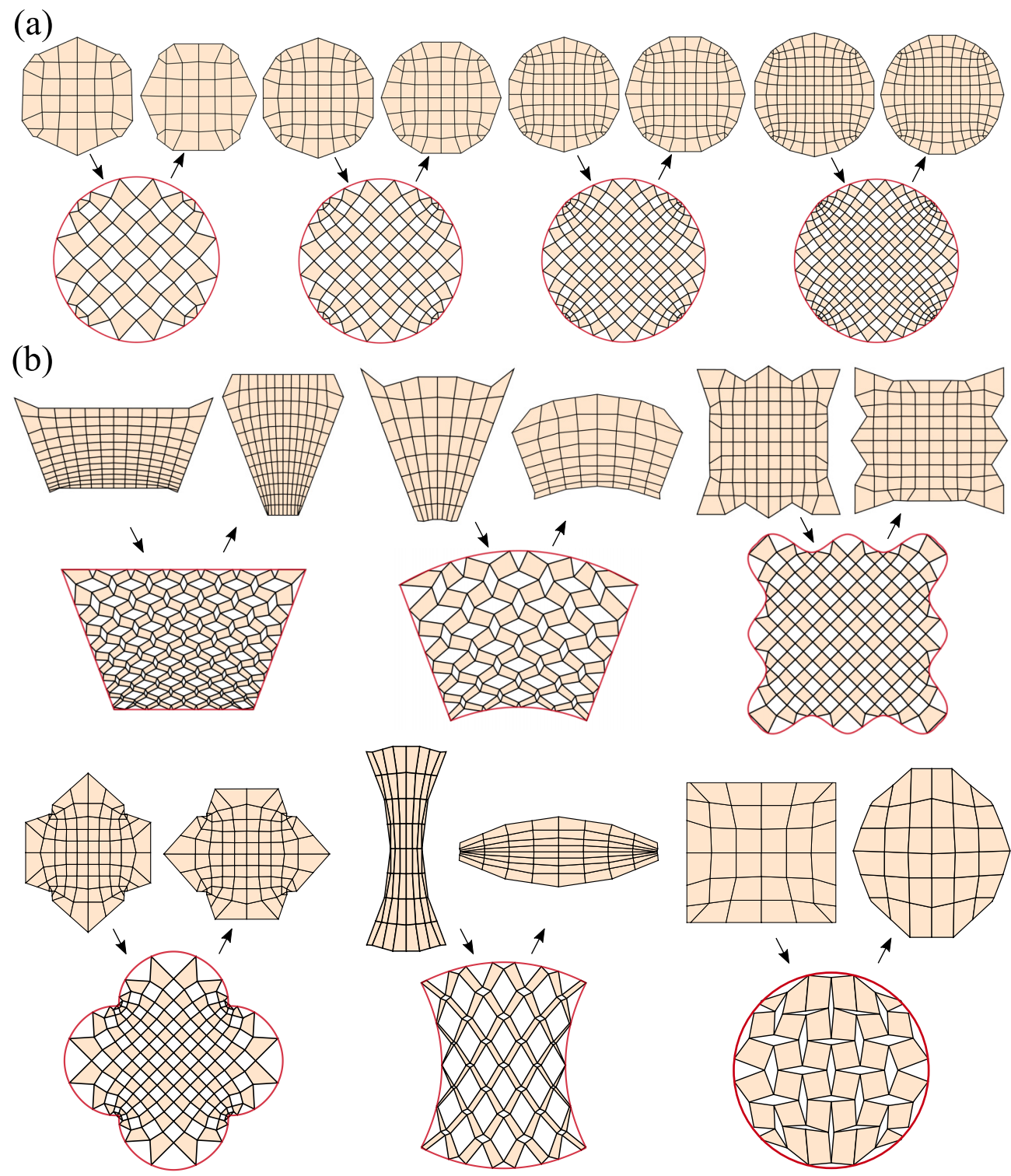

FIG. 9. More examples of reconfigurable, rigid-deployable kirigami patterns with different prescribed target shape. (a) An example of reconfigurable, rigid-deployable quad kirigami patterns at different resolutions. All four patterns can be deployed to approximate a prescribed circle. (b) Even with the additional rigid-deployability constraint, our framework is still capable of producing a large variety of patterns that exhibit highly nontrivial shape change throughout the deployment and contraction process and approximate different target deployed shape.

rigid-deployable example analogous to the structure in Fig. 3(a). Figure 10 shows several reconfigurable, rigiddeployable quad kirigami patterns obtained by solving the optimization problem in the two contracted spaces directly.

\section{EXTENSIONS}

While we have primarily focused on the quad kirigami patterns so far, other bases such as the kagome (triangle-based) kirigami patterns may also be used to construct reconfigurable kirigami patterns. The key is to identify the reconfigurability constraints analogous to Eqs. (3) and (4) for achieving another closed and compact state for the kagome pattern [Fig. 11(a)]. In particular, at each hexagonal negative space, there are two sets of edge length constraints (see the red and blue dotted lines) to be satisfied. Consequently, all six edges of each hexagonal negative space must be equal in length in any reconfigurable kagome kirigami pattern. Figure 11(b) shows a reconfigurable kagome pattern obtained by our framework and the deployment and contraction process.

Besides, so far we have limited ourselves to planar kirigami patterns. We now show how to extend our approach to produce three-dimensional kirigami-based reconfigurable tubular structures that morph from one contracted configuration into another contracted configuration. To achieve these designs, we introduce cuts into a given target tubular shape into patches and isometrically unfold them onto the plane. We then apply our constrained optimization framework to produce a reconfigurable kirigami pattern for each planar shape, with the periodicity of the boundaries corresponding to the cuts 
(a)
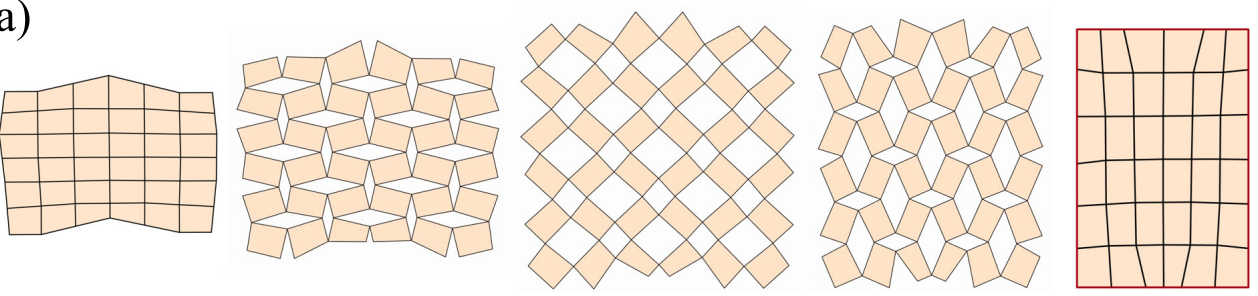

(b)
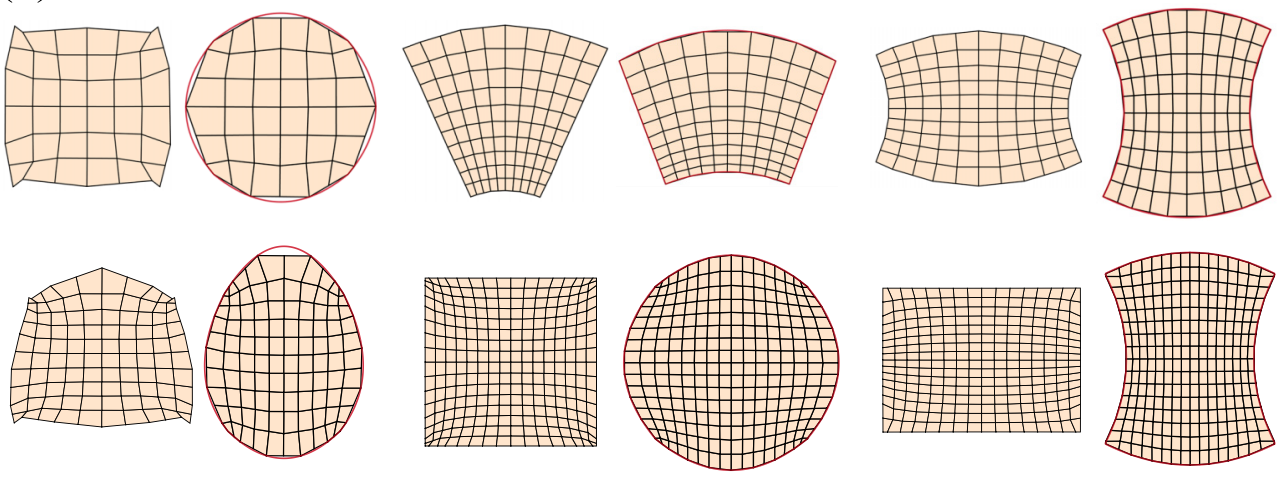

FIG. 10. More examples of reconfigurable, rigid-deployable kirigami patterns obtained by directly solving the optimization problem in the two contracted spaces. For each pattern, one of the contracted states is enforced to approximate a prescribed shape. (a) An example with the two contracted states and several intermediate deployed states. (b) More examples obtained by this approach, each exhibiting a significant shape change in between the two contracted states.

enforced. Finally, the patterns are mapped back to the threedimensional space to form a reconfigurable tubular structure. In Fig. 12(a), we show a three-dimensional kirigami-based reconfigurable tubular structure that morphs from one contracted configuration into another contracted configuration. Figure 12(b) shows another reconfigurable tubular structure achieved by this approach. When compared to the one in
Fig. 12(a), this structure exhibits a smaller axial expansion but a more nonuniform radial change throughout the deployment and contraction process. More complex reconfigurable tubular structures can then be constructed using multiple copies of the patterns obtained by the optimization framework [Fig. 12(c)].

We remark that here we only consider a target 3D tubular shape with zero Gaussian curvature so that we can

(a)
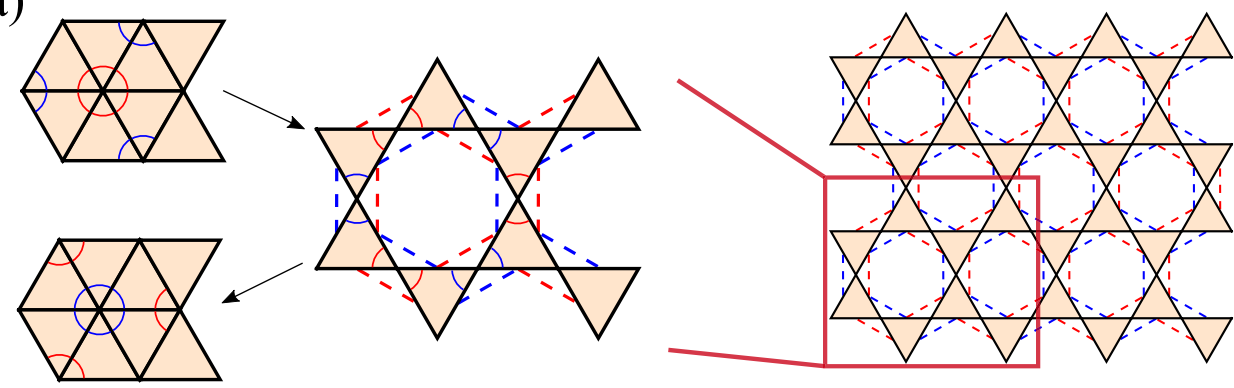

(b)

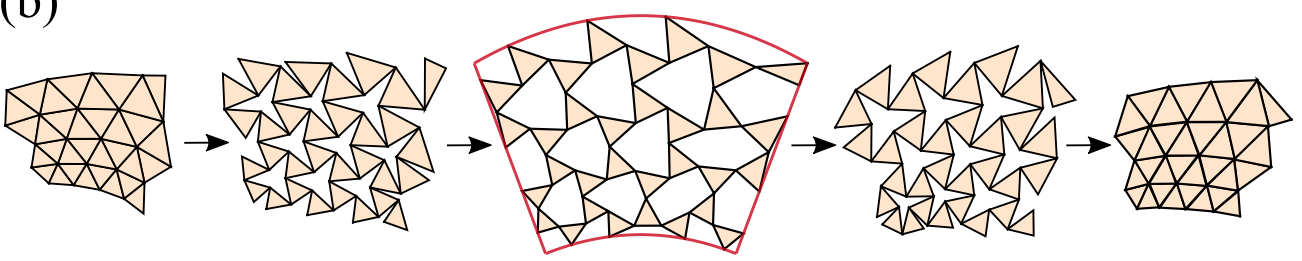

FIG. 11. Extending our framework for producing reconfigurable kagome kirigami patterns. (a) The reconfigurability constraints for the kagome pattern. The red and blue dotted lines indicate the ordinary and dual edge correspondences respectively, and the red and blue arcs indicate the ordinary and dual angle correspondences respectively. (b) The deployment and contraction of a reconfigurable kagome kirigami pattern. 
(a)

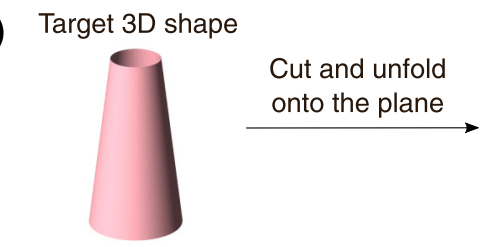

Final reconfigurable tubular structure
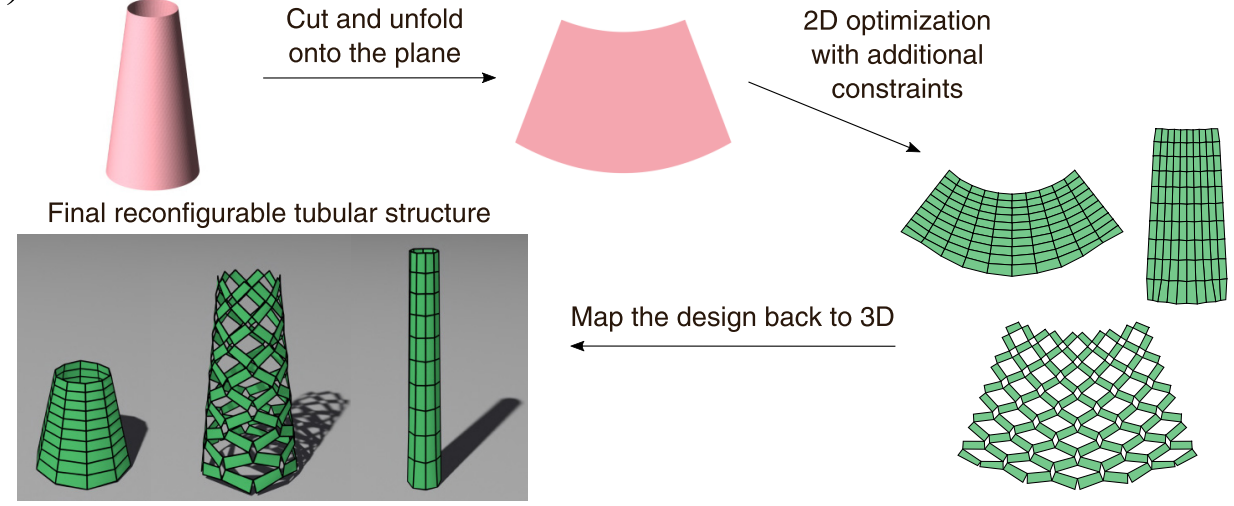

Map the design back to 3D
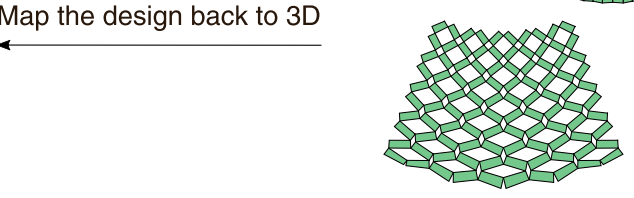

(b)

(c)
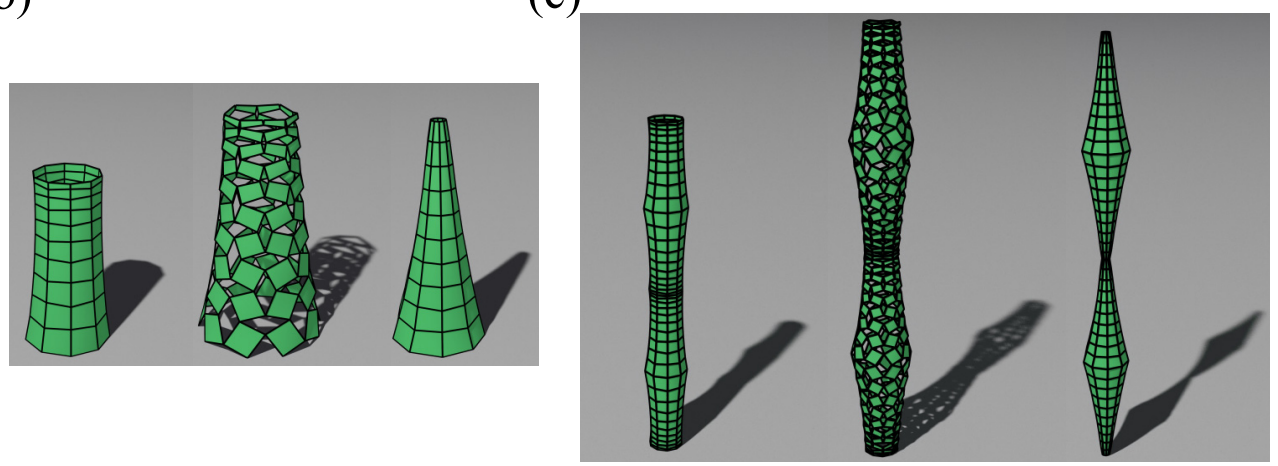

FIG. 12. Extending our framework for producing reconfigurable tubular structures. (a) Our framework can be extended for creating reconfigurable tubular structures which morph from one three-dimensional contracted configuration into another three-dimensional contracted configuration. Given a target three-dimensional (3D) shape, we first cut and unfold it onto the plane. We then solve the 2D constrained optimization problem with an additional periodic boundary constraint to produce a reconfigurable kirigami pattern for the planar shape. We can then map the design back to 3D to form a reconfigurable tubular structure. (b) Another reconfigurable tubular structure with a more nonuniform radial change throughout the deployment and contraction process. (c) A more complex reconfigurable tubular structure produced by assembling four copies of the pattern in (b).

isometrically unfold it onto the plane after introducing a cut to get a target 2D shape for the optimization. As the target tubular shape is with zero Gaussian curvature, the deployed configuration we obtain should also be with zero Gaussian curvature in theory. However, note that mapping the $2 \mathrm{D}$ result back to the 3D space requires interpolating the 3D node positions based on their 2D coordinates, which may involve small numerical error that makes the tubular structure slightly curved [as we can observe in the middle of Figs. 12(b) and 12(c)]. As for the two contracted configurations of the tubular structure, note that they are not necessarily with zero Gaussian curvature. Analogous to origami structures, these closed and compact contracted configurations in three dimensions satisfy the angle sum constraints at every vertex but the dihedral angles between the tiles are not necessarily $\pi$. Besides, as the rigid-deployability theory for the $3 \mathrm{D}$ case is less clear, here we do not include the rigid-deployability constraints in the $2 \mathrm{D}$ optimization problem. The resulting tubular structures may require elastic folding throughout the deployment and contraction process.

\section{DISCUSSION AND CONCLUSION}

While many prior works have studied the geometry, topology, and physics of kirigami patterns, the ability of achieving multiple closed and compact contracted configurations has not been understood. In this paper, we have taken the first step to explore the possibility of such compact reconfigurable design and show that one can achieve reconfigurability in addition to the shape constraints we introduced in our prior framework [6]. We have further shown that it is possible to enforce rigid-deployability in the inverse design framework via an additional set of geometric constraints. All together, our approach exploits the duality in kirigami patterns to create a class of reconfigurable kirigami patterns and planar mechanisms with multiple closed and compact configurations. Just as flat-foldability and rigid-foldability of origami [26] opened the way for understanding and extending origami designs, perhaps their natural analogs in kirigami, viz. contractibility and rigid-deployability, that we have uncovered here might pave the way for a range of art-inspired mathematics, science, and engineering.

\section{ACKNOWLEDGMENTS}

This work was supported in part by the National Science Foundation under Grants No. DMS-2002103 (G.P.T.C.), No. DMR-2011754 (L.M.), No. DMR-1922321 (L.M.), and No. EFRI-1830901 (L.M.), and the Harvard Quantitative Biology Initiative and the NSF-Simons Center for Mathematical 


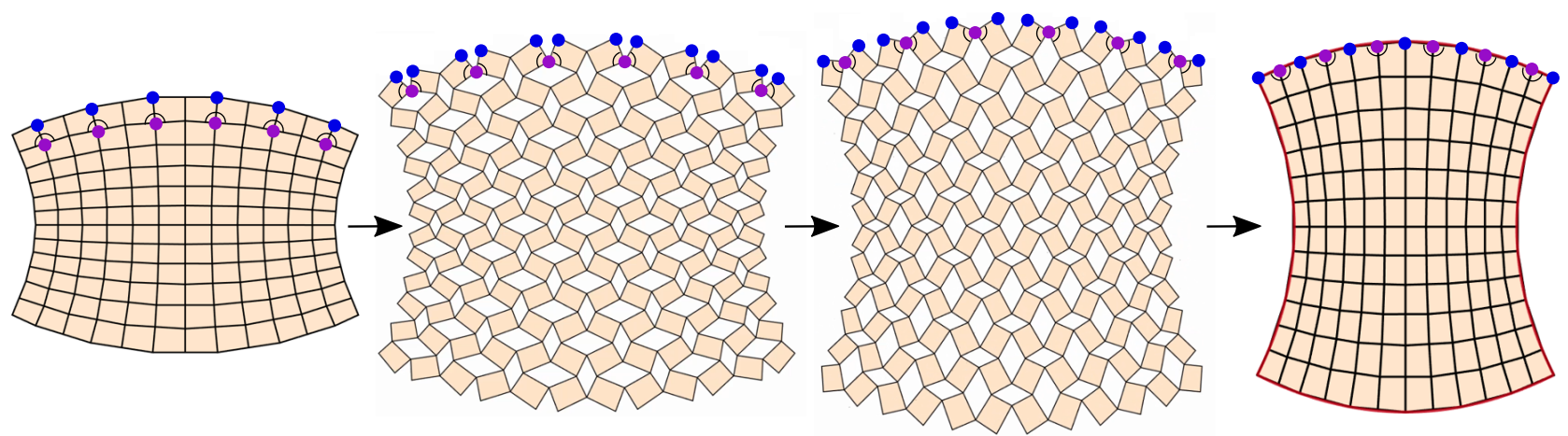

FIG. 13. An example of optimizing the boundary shape of the reconfigured contracted state. Note that only half of the boundary nodes at the reconfigured contracted state (see the blue nodes at the top boundary) can be enforced to lie on the target shape (the red closed curve) precisely. One reason is that the angle sum at the remaining half of the boundary nodes at the reconfigured contracted state (see the purple nodes at the top boundary) is constrained by the rigid-deployability constraints. Another reason is that those purple nodes come from the interior of the initial contracted state, which are constrained by the angle sum constraints and reconfigurable angle sum constraints therein. Enforcing them to lie on the target curve will introduce additional conditions on the relevant angles, making the problem overconstrained. Nevertheless, increasing the resolution of the kirigami pattern can help achieve a better approximation of the target shape even if only half of the boundary nodes in the reconfigured contracted state are controlled.

and Statistical Analysis of Biology at Harvard, Award No. 1764269 (G.P.T.C., L.M.).

\section{APPENDIX A: DEPLOYMENT ENERGETICS}

To study the deployment energetics of the reconfigurable kirigami patterns, we consider a linear spring model similar to the model in [6]. Let $\mathbf{x}_{1}(t), \mathbf{x}_{2}(t), \ldots, \mathbf{x}_{n}(t)$ be the coordinates of the nodes in a reconfigurable kirigami pattern at time $t \in$ $[0,1]$, where $\left\{\mathbf{x}_{i}(0)\right\}_{i=1}^{n}$ and $\left\{\mathbf{x}_{i}(1)\right\}_{i=1}^{n}$ correspond to the two contracted configurations. We introduce a linear spring along every edge and every diagonal of the tiles and consider the following energy:

$$
\begin{aligned}
& E(t) \\
& =\sum_{i, j:[i, j] \text { is an edge or a diagonal }}\left(\frac{\left\|\mathbf{x}_{i}(t)-\mathbf{x}_{j}(t)\right\|-l_{i j}}{l_{i j}}\right)^{2},
\end{aligned}
$$

where $l_{i j}$ is the rest length of the spring at $[i, j]$. To get the deployment path, we start with a contracted configuration (obtained by the constrained optimization framework) and continuously pull two opposite nodes towards their

\section{(a)}
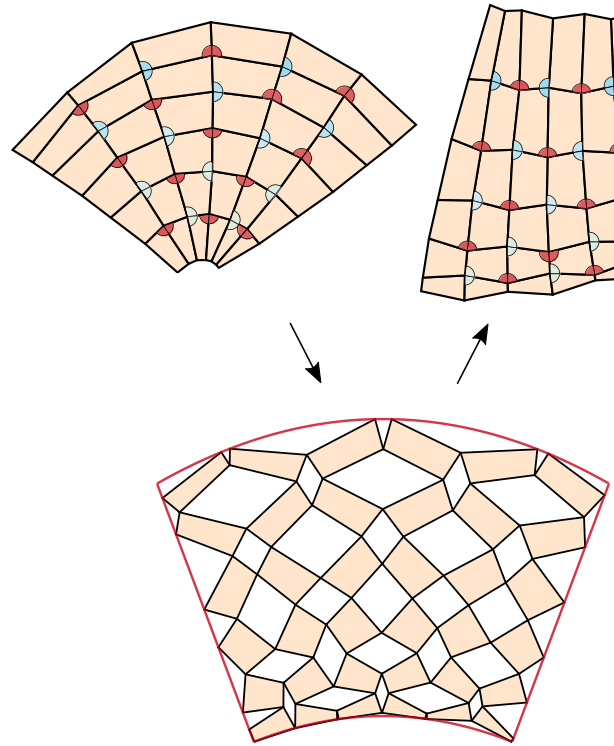

(b)
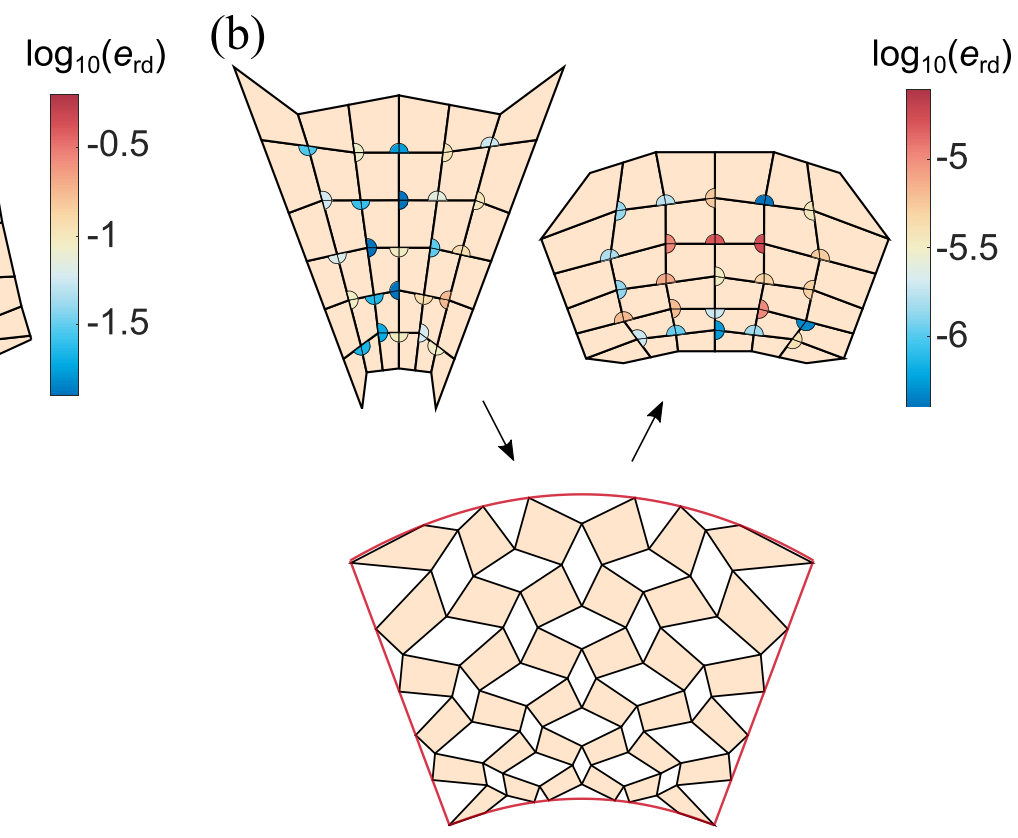

FIG. 14. Rigid-deployability constraint violation. (a) A reconfigurable kirigami pattern obtained by our proposed design framework without imposing the rigid-deployability constraints [Eq. (9)]. (b) A reconfigurable kirigami pattern obtained by our proposed design framework with the rigid-deployability constraints included in the optimization process. In each example, the color of the angles in the first contracted state represents the value of $\log _{10}\left[e_{\mathrm{rd}}\left(\alpha_{1}, \alpha_{2}\right)\right]$, and the color of the angles in the second contacted state represents the value of $\log _{10}\left[e_{\mathrm{rd}}\left(\beta_{1}, \beta_{2}\right)\right]$, where $\alpha_{1}, \alpha_{2}, \beta_{1}, \beta_{2}$ are the angles described in Eq. (9). 
corresponding positions in the target deployed configuration (also obtained by the constrained optimization framework). To remove the global translations and rotations of the model, we focus on symmetric patterns and pull two opposite nodes along the axis of symmetry. At each time point, we solve for the intermediate deployed configuration by minimizing Eq. (A1) subject to the positional constraints of the two nodes. After reaching the target deployed configuration, we repeat the process by pulling two nodes towards their corresponding positions in the second contracted configuration (obtained by the constrained optimization framework).

As guaranteed by the constrained optimization framework, the energy is zero at both contracted configurations and the target deployed configuration for any reconfigurable kirigami pattern. This results in a multistable energy landscape. If the rigid-deployability constraints are further enforced, all tiles will undergo no deformation throughout the deployment process and hence $E=0$ at all time $t$ theoretically.

\section{APPENDIX B: CONSTRAINED OPTIMIZATION IN THE TWO CONTRACTED SPACES}

As mentioned in the main text, in case it is more desirable to design a reconfigurable kirigami pattern that achieves a target reconfigured contracted shape without caring about how the deployed states look like, we can formulate a constrained optimization in the two contracted spaces directly.

Denote the initial contracted state by $S_{1}$ and the reconfigured contracted state by $S_{2}$. Let $\left\{\mathbf{x}_{i}\right\}_{i=1}^{n}$ and $\left\{\mathbf{y}_{i}\right\}_{i=1}^{n}$ be the nodes in $S_{1}$ and $S_{2}$ respectively. Suppose we would like $S_{2}$ to approximate a prescribed target shape (such as a circle). To solve for a valid reconfigurable and rigid-deployable kirigami pattern that satisfies the requirement, we consider the optimization over all $2 n$ nodes $\mathbf{x}_{1}, \ldots, \mathbf{x}_{n}, \mathbf{y}_{1}, \ldots, \mathbf{y}_{n}$ (i.e., $4 n$ coordinates in total) with the following constraints:

(1) Corresponding edges in $S_{1}$ and $S_{2}$ should be equal in length.

(2) Corresponding angles in $S_{1}$ and $S_{2}$ should be equal.

(3) Rigid-deployability constraints for the angles in $S_{1}$ (i.e., $\alpha_{1}+\alpha_{2}=\alpha_{3}+\alpha_{4}=\beta_{1}+\beta_{2}=\beta_{3}+\beta_{4}=\pi$ as discussed in the main text).

(4) Target shape matching constraints for half of the boundary nodes in $S_{2}$.

(5) (Optional) Boundary shape matching constraints for half of the boundary nodes in $S_{1}$.

The constraints (i) and (ii) ensures the consistency (in terms of the edge lengths and the angles) between the two contracted states. Note that the two constraints are different from the original edge length constraints (which are about edge pairs in the same deployed state) and the original angle sum constraints (as the angle sum is automatically $2 \pi$ in the contracted states here). The constraints (iii) guarantee the rigid-deployability/contractibility by optimizing the angles in one of the contracted states [the constraint (ii) will change the angles in the other contracted state accordingly]. The constraints (iv) and (v) control the boundary shape of the two contracted states.

For (iv) and (v), note that the shape matching constraints can only be enforced for the half of the boundary nodes which always remain to be at the boundary throughout the

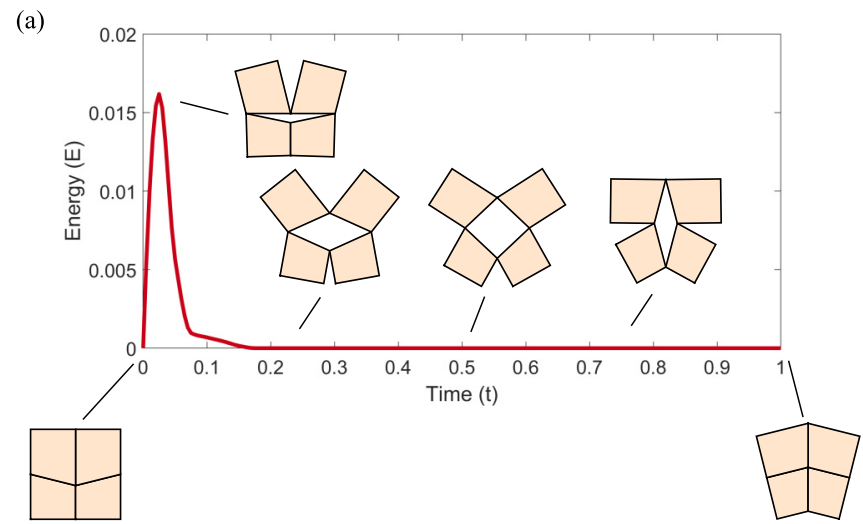

(b)

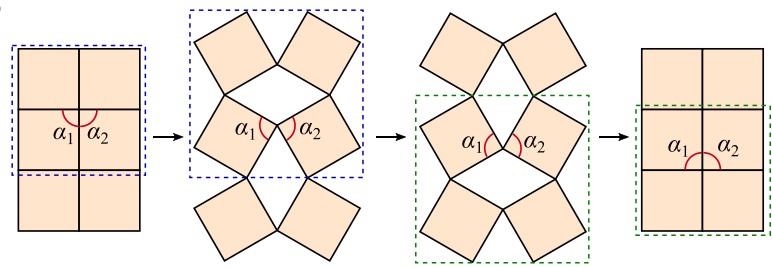

FIG. 15. Possibility of achieving other energy landscapes. (a) A $2 \times 2$ reconfigurable kirigami pattern that only satisfies half of the rigid-deployability constraints. More specifically, we have $\beta_{1}+\beta_{2}=$ $\beta_{3}+\beta_{4}=\pi$ in Eq. (9) while $\alpha_{1}+\alpha_{2} \neq \pi$. For this pattern, the energy landscape contains only one energy bump. (b) For a $3 \times 2$ reconfigurable kirigami pattern, note that the angles $\alpha_{1}, \alpha_{2}$ are related to the geometrical frustration in opening the negative space in one unit cell (highlighted by the blue dotted lines). After the deployment and contraction process, one can see that these angles are also related to the geometrical frustration in recontracting the negative space in another unit cell (highlighted by the green dotted lines). This shows that the constraints involving $\alpha_{i}$ 's and the constraints involving $\beta_{i}$ 's in some neighboring cells are related, and hence one cannot eliminate one of the energy bumps by simply enforcing only half of Eq. (5).

deployment and contraction from $S_{1}$ to $S_{2}$ (see the blue nodes in Fig. 13). One reason is that if the rigid-deployability constraints are enforced, the angle sum at the remaining half of the boundary nodes (see the purple nodes) in the two contracted states must be $\pi$ at the first contracted state. After those purple nodes move to the boundary at the reconfigured contracted state, the angle sum at each of them should still be $\pi$ and hence the two boundary edges incident to each of them always form a straight line. Therefore, those nodes cannot fit an arbitrary given shape at the second contracted state precisely. Moreover, even if the pattern is not enforced to be rigid deployable, it is still impossible to control the shape at some boundary nodes precisely. The reason is that the reconfigurable angle sum constraint at those purple nodes at the top boundary will propagate to the bottom boundary via a sequence of angle sum constraints and reconfigurable angle sum constraints at the interior. To fit some curved shape such as a circle, there must be some angle condition that the angles at those purple nodes should satisfy (e.g., the angle sum at such a node must be less than $\pi$ in order to approximate the curvature of the circle), and such conditions at one purple node will limit the angle sum and hence the shape at its opposite boundary. Nevertheless, by increasing the resolution of 
(a)

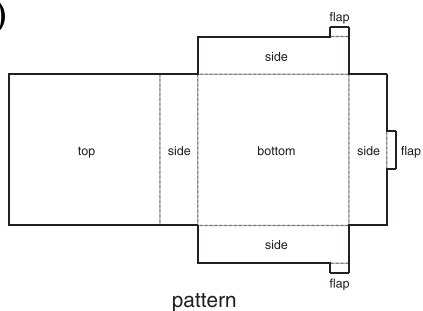

pattern
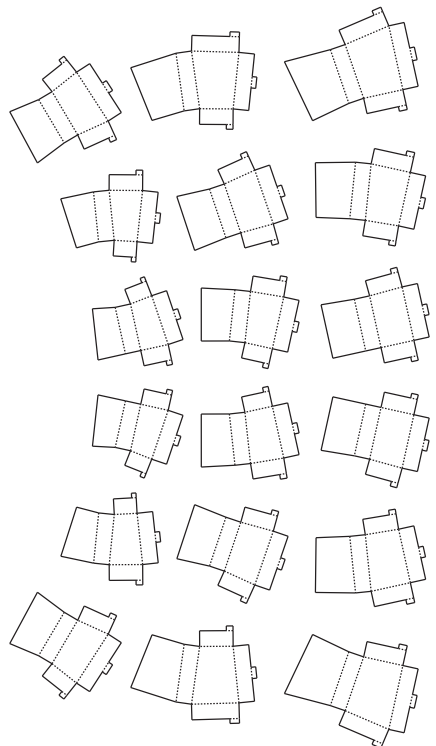
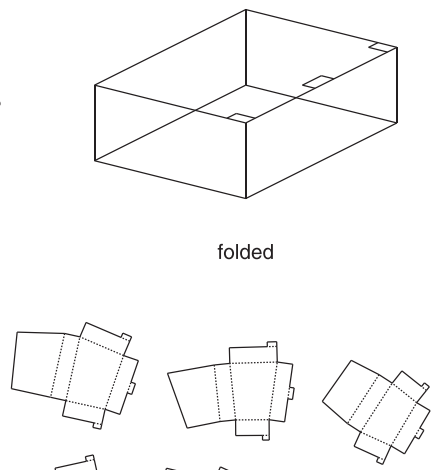

folded
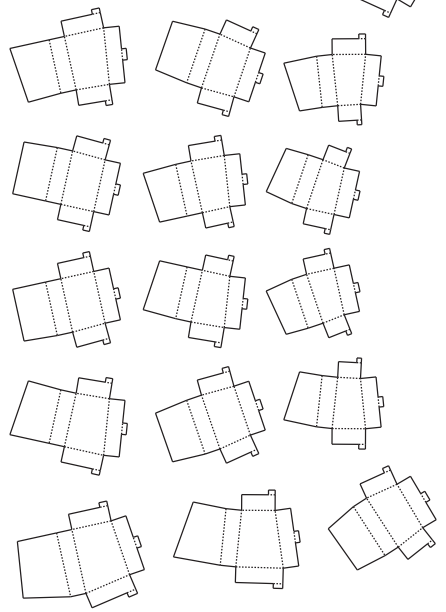

(b)
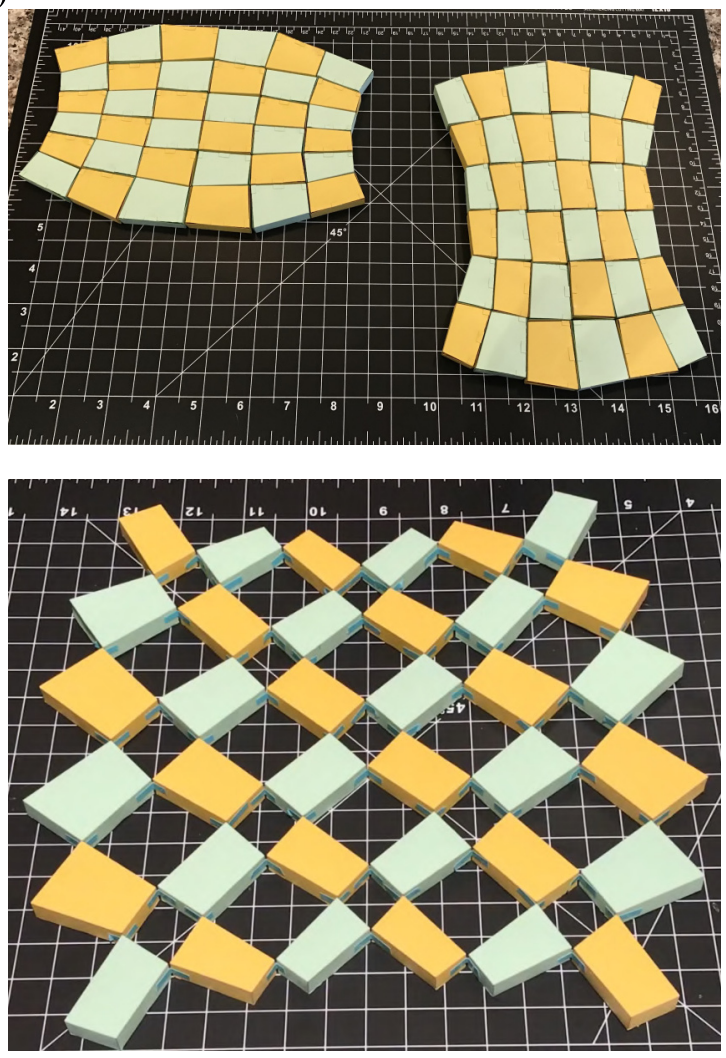

FIG. 16. A physical paper model of the reconfigurable and rigid-deployable kirigami pattern in Fig. 7(a). (a) We consider each tile as a polyhedron with certain thickness and create a planar layout pattern with some extra small faces to give height to the folded pieces. (b) We can then obtain the paper layout patterns by laser cutting. The paper layout patterns are folded and glued to form the tiles (top), which are subsequently assembled using tape to form a deployable structure (bottom).

the kirigami pattern, one can approximate the target boundary shape very well even by enforcing the target shape matching constraints for only half of the boundary nodes.

\section{APPENDIX C: ANALYSIS OF THE PATTERNS}

\section{Quantifying the rigid-deployability constraint violation}

To quantify the nonrigid deployability of different kirigami patterns, we define the rigid-deployability constraint violation by the absolute difference between $\pi$ and the sum of each pair of angles described in the rigid-deployability constraints [Eq. (9)]:

$$
e_{\mathrm{rd}}\left(\alpha_{1}, \alpha_{2}\right)=\left|\pi-\alpha_{1}-\alpha_{2}\right|,
$$

where $\alpha_{1}, \alpha_{2}$ are two corresponding angles in Eq. (9). Figure 14 shows two examples of reconfigurable kirigami patterns with the same target deployed shape. The pattern in Fig. 14(a) is obtained via the constrained optimization without imposing the rigid-deployability constraints, while the one in Fig. 14(b) is obtained with the rigid-deployability constraints imposed. Note that $e_{\mathrm{rd}}$ is significantly smaller for the second example. This can also be observed from the fact that most of the relevant four-bar linkages (the negative spaces) do not form straight lines in the two contracted states in Fig. 14(a), while those in Fig. 14(b) form visually straight lines. More specifically, we have $\operatorname{mean}\left(e_{\mathrm{rd}}\right) \sim 10^{-1}$ for the first example, while mean $\left(e_{\mathrm{rd}}\right) \sim 10^{-6}$ for the second example.

We remark that sometimes the constraint violation for the patterns obtained without enforcing the rigid-deployability constraints can also be small. For example, it can be observed that the contracted negative spaces in the first circle example in Fig. 4(b) also form visually straight lines [with mean $\left(e_{\mathrm{rd}}\right) \sim$ $10^{-4}$ ]. However, in general, if the rigid-deployability constraints are not enforced in the optimization problem, it is expected that the resulting patterns will be with a relatively large $e_{\mathrm{rd}}$ and hence are not rigid deployable. The energy landscape will then be with at least two notable bumps as shown in Fig. 3(c). As discussed in the main text, the violation of the rigid-deployability constraints is related to the changes in the edge lengths in a negative space/four-bar linkage needed for passing from the contracted state to the deployed states. Therefore, the energy bumps decrease continuously as the geometry of the kirigami pattern approaches the rigid-deployable case.

\section{More variations}

Besides the multistable energy landscape of the reconfigurable kirigami patterns and the zero energy landscape of the reconfigurable, rigid-deployable kirigami patterns, one may be interested in achieving other energy landscapes. For a simple $2 \times 2$ quad unit cell, note that the first bump in the 
energy landscape is related to the violation of the first half of Eq. (9) (i.e., $\alpha_{1}+\alpha_{2}=\alpha_{3}+\alpha_{4}=\pi$ ), which is about the geometrical frustration encountered when opening the negative spaces from the first closed and compact configuration. Similarly, the second energy bump is related to the violation of the latter half of Eq. (5) (i.e., $\beta_{1}+\beta_{2}=\beta_{3}+\beta_{4}=\pi$ ). Therefore, it is natural to ask whether one can achieve an energy landscape without one of the two energy bumps by enforcing only half of Eq. (5). Indeed, it is possible to obtain a $2 \times 2$ reconfigurable quad kirigami pattern with such an energy landscape. As shown in Fig. 15(a), if the pattern only satisfies $\beta_{1}+\beta_{2}=\beta_{3}+\beta_{4}=\pi$ but not $\alpha_{1}+\alpha_{2}=\alpha_{3}+\alpha_{4}=\pi$, then the resulting energy landscape only contains one energy bump. However, for patterns with more tiles, we note that the constraints involving $\alpha_{i}$ 's and the constraints involving $\beta_{i}$ 's in some neighboring cells are actually related to each other. As shown in Fig. 15(b), the two angles $\alpha_{1}, \alpha_{2}$ are related to the first contracted negative space in the $2 \times 2$ unit cell formed by the top four tiles (blue dotted lines). After the deployment and contraction process, one can see that these angles are in fact also related to the reconfigured contracted negative space in the $2 \times 2$ unit cell formed by the bottom four tiles (green dotted lines). Therefore, if we enforce the first half of Eq. (9) in one unit cell, then it automatically enforces the second half of Eq. (9) for some other unit cell. Hence, for larger patterns, it is impossible to eliminate only one of the two energy bumps by simply enforcing half of the rigid-deployability constraints.

\section{APPENDIX D: PHYSICAL MODELS}

The designed reconfigurable kirigami patterns can be physically realized. Besides the acrylic plastic models and wooden models shown in the main text, we show in Fig. 16 an alternative method to produce physical models with folded paper pieces.
[1] J. N. Grima, A. Alderson, and K. E. Evans, Negative Poisson's ratios from rotating rectangles, Comput. Methods Sci. Technol. 10, 137 (2004).

[2] H. Mitschke, V. Robins, K. Mecke, and G. E. Schröder-Turk, Finite auxetic deformations of plane tessellations, Proc. R. Soc. A 469, 20120465 (2013).

[3] S. Shan, S. H. Kang, Z. Zhao, L. Fang, and K. Bertoldi, Design of planar isotropic negative Poisson's ratio structures, Extreme Mech. Lett. 4, 96 (2015)

[4] A. Rafsanjani and D. Pasini, Bistable auxetic mechanical metamaterials inspired by ancient geometric motifs, Extreme Mech. Lett. 9, 291 (2016).

[5] B. G.-G. Chen, B. Liu, A. A. Evans, J. Paulose, I. Cohen, V. Vitelli, and C. D. Santangelo, Topological Mechanics of Origami and Kirigami, Phys. Rev. Lett. 116, 135501 (2016).

[6] G. P. T. Choi, L. H. Dudte, and L. Mahadevan, Programming shape using kirigami tessellations, Nat. Mater. 18, 999 (2019).

[7] S. Chen, G. P. T. Choi, and L. Mahadevan, Deterministic and stochastic control of kirigami topology, Proc. Natl. Acad. Sci. USA 117, 4511 (2020).

[8] C. Jiang, F. Rist, H. Pottmann, and J. Wallner, Freeform quadbased kirigami, ACM Trans. Graph. 39, 1 (2020).

[9] M. K. Blees, A. W. Barnard, P. A. Rose, S. P. Roberts, K. L. McGill, P. Y. Huang, A. R. Ruyack, J. W. Kevek, B. Kobrin, D. A. Muller et al., Graphene kirigami, Nature (London) 524, 204 (2015)

[10] T. C. Shyu, P. F. Damasceno, P. M. Dodd, A. Lamoureux, L. $\mathrm{Xu}$, M. Shlian, M. Shtein, S. C. Glotzer, and N. A. Kotov, A kirigami approach to engineering elasticity in nanocomposites through patterned defects, Nat. Mater. 14, 785 (2015).

[11] R. M. Neville, F. Scarpa, and A. Pirrera, Shape morphing kirigami mechanical metamaterials, Sci. Rep. 6, 31067 (2016).

[12] P. Celli, C. McMahan, B. Ramirez, A. Bauhofer, C. Naify, D. Hofmann, B. Audoly, and C. Daraio, Shape-morphing architected sheets with non-periodic cut patterns, Soft Matter 14, 9744 (2018).

[13] M. Konaković-Luković, J. Panetta, K. Crane, and M. Pauly, Rapid deployment of curved surfaces via programmable auxetics, ACM Trans. Graph. 37, 1 (2018).
[14] A. Rafsanjani, Y. Zhang, B. Liu, S. M. Rubinstein, and K. Bertoldi, Kirigami skins make a simple soft actuator crawl, Sci. Robot. 3, eaar7555 (2018)

[15] J. I. Lipton, R. MacCurdy, Z. Manchester, L. Chin, D. Cellucci, and D. Rus, Handedness in shearing auxetics creates rigid and compliant structures, Science 360, 632 (2018).

[16] Y. Yang and Z. You, Geometry of transformable metamaterials inspired by modular origami, J. Mech. Robot. 10, 021001 (2018).

[17] M. Stavric and A. Wiltsche, Geometrical elaboration of auxetic structures, Nexus Netw. J. 21, 79 (2019).

[18] Z. You and S. Pellegrino, Foldable bar structures, Int. J. Solids Struct. 34, 1825 (1997).

[19] D. Mao, Y. Luo, and Z. You, Planar closed loop double chain linkages, Mech. Mach. Theory 44, 850 (2009).

[20] J. M. McCarthy and G. S. Soh, Geometric Design of Linkages, Vol. 11 (Springer, New York, 2010).

[21] Z. You and Y. Chen, Motion Structures: Deployable Structural Assemblies of Mechanisms (CRC Press, New York, 2011).

[22] T. G. Nelson, T. K. Zimmerman, S. P. Magleby, R. J. Lang, and L. L. Howell, Developable mechanisms on developable surfaces, Sci. Robot. 4, eaau5171 (2019).

[23] See Supplemental Material at http://link.aps.org/supplemental/ 10.1103/PhysRevResearch.3.043030 for the videos of the deployment of several numerical and physical models produced by our framework.

[24] G. P.-T. Choi and L. M. Lui, A linear formulation for disk conformal parameterization of simply-connected open surfaces, Adv. Comput. Math. 44, 87 (2018).

[25] T. W. Meng, G. P.-T. Choi, and L. M. Lui, TEMPO: Featureendowed Teichmüller extremal mappings of point clouds, SIAM J. Imaging Sci. 9, 1922 (2016).

[26] T. Kawasaki, On the relation between mountain-creases and valley-creases of a flat origami, in Proceedings of the 1st International Meeting on Origami Science and Technology, edited by H. Huzita (Universita di Padova, Ferrara, Italy, 1989), pp. 229-237. 